\title{
BRMS1 $1_{51-98}$ and BRMS1 $1_{51-84}$ are crystal oligomeric coiled-
}

\section{coils with different stoichiometry, which behave as disordered}

\section{proteins in solution}

Mercedes Spínola-Amilibia ${ }^{1,2}$, José Rivera ${ }^{3}$, Miguel Ortiz-Lombardía ${ }^{4}$, Antonio Romero $^{2}$, José L. Neira ${ }^{5,6, *}$ and Jerónimo Bravo ${ }^{1, *}$

${ }^{1}$ Instituto de Biomedicina de Valencia (IBV-CSIC), C/ Jaime Roig 11, 46010 Valencia, Spain;

${ }^{2}$ Departamento de Biología Físico-Química, Centro de Investigaciones Biológicas (CIBCSIC), C/ Ramiro de Maeztu 9, 28040 Madrid, Spain.

${ }^{3}$ Department of Epidemiology, Atherothrombosis and Imaging, Spanish National Cardiovascular Research Center (CNIC), 28029, Madrid, Spain.

${ }^{4}$ Architecture et Fonction des Macromolécules Biologiques, UMR6098, CNRS, Aix-Marseille Université, Case 932, 163 Avenue de Luminy, 13288, Marseille cedex 9, France.

${ }^{5}$ Instituto de Biología Molecular y Celular, Universidad Miguel Hernández, Elche (Alicante);

${ }^{6}$ Instituto de Biocomputación y Física de Sistemas Complejos, Zaragoza; Spain.

Short title: Oligomeric BRMS1 proteins

*Corresponding authors' addresses: José L. Neira, Instituto de Biología Molecular y Celular, Universidad Miguel Hernández, Avda. del Ferrocarril s/n, 03202, Elche (Alicante), Spain. Email: jlneira@umh.es. Jerónimo Bravo, Department of Genomics and Proteomics. Instituto de Biomedicina de Valencia (IBV-CSIC), C/ Jaime Roig 11, 46010 Valencia, Spain: Email: jbravo@ibv.csic.es.

Keywords: X-ray; coiled-coils; NMR, BRMS1; biophysical features. 
Abbreviations used: AUC, analytical ultracentrifugation; ANS, 1-anilino-8-naphtalene sulfonate; BRMS1, Breast Cancer Metastasis Suppressor 1; D, diffusion coefficient; $C D$, Circular Dichroism; DOSY, Diffusion Ordered Spectroscopy; $\mathrm{GdmCl}$, guanidinium hydrochloride; RMSD, root mean square deviation; TFE, [2,2,2]-trifluoroethan-1-ol; NES, nuclear export signal; TLS, translation/libration/screw; TSP, sodium trimethylsilyl-[2,2,3,3${ }^{2} \mathrm{H}_{4}$ ]-propionate; $T_{m}$, thermal-denaturation midpoint; $U V$, ultraviolet. 


\begin{abstract}
The Breast cancer metastasis suppressor 1 gene suppresses metastasis without affecting the primary tumour growth. Cellular localisation of BRMS1 appears to be important for exerting its effects on metastasis inhibition. We recently described a nucleo-cytoplasmic shuttling for BRMS1 and identified a nuclear export signal within the N-terminal coiled coil. The structure of these regions shows an antiparallel coiled coil capable of oligomerising, which compromises the accessibility to the nuclear export signal consensus residues. We have studied the structural and biophysical features of this region to further understand the contribution of the N-terminal coiled coil to the biological function of BRMS1. We have observed that residues 85 to 98 might be important in defining the oligomerisation state of the BRMS1 N-terminal coiled coil. In addition, we report the presence of a conformational dynamic equilibrium (oligomeric folded species $\leftrightarrow$ oligomeric unfolded) in solution in the BRMS1 N-terminal coiled coil that might facilitate the nuclear export of BRMS1 to the cytoplasm.
\end{abstract}




\section{INTRODUCTION}

Breast cancer metastasis suppressor 1 (BRMS1) functions as a metastasis suppressor gene that inhibits metastasis in breast carcinoma, melanoma, ovarian carcinoma and nonsmall cell lung carcinoma, without preventing primary tumor growth ${ }^{1}$. BRMS1 is epigenetically regulated since it has been shown that in tumorigenic and metastatic cell lines, a $\mathrm{CpG}$ island in the promoter region is methylated, leading to the suppression of its expression $^{2}$. The presence of BRMS1 does not entirely inhibit a single step of the metastatic cascade but it blocks $80-90 \%$ of metastasis in mouse models by intervening at different stages of the metastatic progression. BRMS1 is capable of performing this wide range of cellular functions by regulating gene expression ${ }^{3 ; 4 ; 5 ; 6 ; 7}$ since it interacts with SIN3:HDAC chromatin remodelling complexes ${ }^{4 ; 8 ; 9}$ and inhibits NFKB activity ${ }^{3 ; 10}$. BRMS1 sequence contains two coiled coil regions that participate in several interactions with different cellular partners in order to perform BRMS1 cellular functions ${ }^{4 ; 11 ; 12}$. BRMS1 is mostly localised to the nucleus although we have reported a nuclear-cytoplasm shuttling due to functional nuclear localisation and nuclear export signals in the BRMS1 sequence ${ }^{13}$. This observation suggests that BRMS1 activity might be at least partially modulated by cellular compartmentalisation.

Despite its clear role in metastasis suppression, molecular characterization of BRMS1, including structural studies, have remained largely elusive. We have recently determined the structure of BRMS1 N-terminal coiled coil ${ }^{14}$. BRMS1 residues 51 to 98 form an antiparallel coiled coil that homo-oligomerizes in a hexameric conformation as it was confirmed by hydrodynamic experiments ${ }^{14}$. The crystallised coiled coil also includes BRMS1 residues 7491 that we previously identified as a weak functional Nuclear Export Signal (NES) ${ }^{13}$. The oligomeric organisation of the N-terminal coiled coil does not allow accessibility of the key residues that define the NES. Therefore, the N-terminal coiled coil should disassemble prior to performing its nuclear export function. Cellular localisation is particularly relevant since it has been shown that a BRMS1 localization shift to the cytoplasm is associated with highly proliferative ER-negative breast cancers ${ }^{15}$ and changes in melanoma progression ${ }^{16}$. Moreover, 
we have previously reported the crystallization of BRMS1 including residues 51 to $84^{17}$ and did not detect the hexameric oligomer association shown for BRMS1 $1_{51-98}$.

To further understand the contribution of the N-terminal coiled coil to the biological function of BRMS1 and analyse its role in oligomerisation, we have solved the X-ray structure of BRMS1 residues 51 to 84; moreover, we have studied the conformational features of both BRMS1 $1_{51-84}$ and BRMS1 $1_{51-98}$ in solution to provide clues on the importance of the additional residues (Q85-A98) into the oligomerisation states observed in the X-ray structures. By studying the biophysical properties of both BRSMSs species, we aim to determine its stability and how the additional polypeptide region affects the properties of the chain in solution.

We have observed that residues Q85 to A98 are important in defining the oligomerisation state of the BRMS1 N-terminal coiled coil, despite not being directly involved in the BRMS1 $1_{51-98}$ hexameric conformation. The biophysical characterization in aqueous solution shows $\mathrm{pH}$-dependent structural changes possibly modulated by the additional polypeptide region in BRMS1 $1_{51-98}$. Furthermore, the study of the conformational stability by $\mathrm{pH}$, chemical denaturants and temperature of both BRMS1 fragments suggests the presence of a dynamic conformational equilibrium between different oligomeric states.

\section{RESULTS}

BRMS1 $_{51-84}$ crystal structure

The human BRMS1 $1_{51-84}$ mercury derivatised crystals belong to the $\mathrm{C} 222_{1}$ space group with unit cell dimensions $a=42.41 \AA, b=191.40 \AA$ and $c=71.87 \AA$ and $\alpha=90.0^{\circ}, \beta=90.0$ ${ }^{\circ}$ and $\gamma=90.0^{\circ}$. Diffraction data were collected and reduced to 2.0 $\AA$. Statistics obtained after data reduction and processing are summarized in Table 1.

(a) BRMS1 $1_{51-84}$ asymmetric unit: The final model contains eight BRMS1 $1_{51-84}$ molecules $(\mathrm{A}-\mathrm{H})$ in the asymmetric unit, bound together in pairs (A-D; B-E; C-H and F-G) by a disulphide bridge through the Cys60 of each monomer (Fig. 1 A), with an average distance 
between sulphur atoms (SG, Fig. 1 A) of $2 \AA .208$ residues, of a total of 280, contained within the asymetric unit, could be modelled, being chains $A$ and $C$ fully built (including BRMS1 sequence from E51 to S84, plus an extra serine at the N-terminus, remaining after cleavage of the His-tag). Twelve aminoacids were traced with side-chain double conformation. One sulphate, one chloride ion, one acetic acid and a total of 153 water molecules were also included in the final model (Table 1).

Most $(98.96 \%)$ of the residues in the final model are located in the preferred region of the Ramachandran diagram, $0.52 \%$ in allowed areas and only the exposed E59 of chain G in an energetically unfavorable region.

(b) BRMS1 $1_{51-84}$ biological unit: The chain pairs A-Asym2 and G-Csym5 (where Asym2 and Csym5 are, respectively, chain A and chain C from symmetry-related molecules in the crystal), are arranged as a left-handed antiparallel coiled coils, as shown in Fig. 1 B and 2. AAsym 2 and G-Csym 5 pairs present a total surface contact area of $1,966.4 \AA^{2}$. The coiled coil is mainly supported by hydrophobic contacts towards the ends and by polar interactions in the central region (Fig. 2).

Similarly to the structure of BRMS $1_{51-98}{ }^{14}$, analysis of the BRMS1 $1_{51-84}$ structure with the program Monster, which checks residue contacts, and Twister, defines a heptad pattern repetition (Fig. 3 A) typical of coiled coils. BRMS1 $1_{51-84}$ crystal structure shows an antiparallel coiled coil including residues E51 to S84, plus one additional Ser (S-1) from the His-tag, after cleavage with protease. Most of the residues located at positions $a$ and $d$ in the heptad pattern are hydrophobic (Val, Leu or Phe), as shown in the Fig. 3 B. These residues are key in the interactions that uphold the coiled coil. The coiled coils in the BRMS1 $1_{51-84}$ crystal structure include two complete and two half-heptad repeats with a stutter prior to S72, as shown in Fig. 3 A. Positions $a$ are occupied by L65, E76 and L83, whereas V61, E68, S72 and F79 fill in positions $d$. Contrary to the trimer of antiparallel coiled coils reported for BRMS1 $1_{51-98}$, the BRMS1 $_{51-84}$ crystal structure does not suggest any oligomerisation state other than the mentioned antiparallel coiled coil. 
We further tested the oligomeric character of BRMS1 $1_{51-84}$ by carrying out DOSY-NMR measurements and AUC experiments. The DOSY experiments suggest that BRMS1 $1_{51-84}$, contains oligomeric species. The determined $D$ is $(1.04 \pm 0.02) \times 10^{-6} \mathrm{~cm}^{2} \mathrm{~s}^{-1}$, which yields a hydrodynamic radius $R_{h}$ of $17.7 \AA$. We can estimate the expected $R_{h}$ for BRMS1 $1_{51-84}$ assuming that it is a monomeric folded protein $\left(R_{h}=(4.75 \pm 1.11) N^{0.29 \pm 0.02}\right.$, where $N$ is the number of residues $^{18}$ ). Under the rough simplification that we can consider the protein as globular, these expressions yield $13 \pm 3 \AA$ for a monomeric protein, and $19 \pm 5$ for a tetrameric species.

The $\mathrm{c}(\mathrm{s})$ profile of the protein obtained in the velocity experiment analysis (Fig. 4 A) shows a single peak at $0.5 \mathrm{mg} / \mathrm{mL}(114.5 \mu \mathrm{M})$ with a sedimentation coefficient of $0.79 \pm 0.1$ S. Sedimentation equilibrium experiments (Fig. 4 B) were also carried out in parallel to determine the molecular weight of BRMS1 $1_{51-84}$. Experimental data were fitted to a model with single species, resulting in a molecular weight of around 9,900 $\pm 30 \mathrm{Da}$ at a concentration of 114.5 $\mu \mathrm{M}$. The residuals of the fitting are shown in Fig. 4 B. For comparison, the calculated molecular weight of a BRMS1 $1_{51-84}$ monomer is 4366.8 Da.

\section{Biophysical characterization of BRMS1 $1_{51-84}$ and BRMS1 $1_{51-98}$}

The X-ray, AUC and DOSY-NMR measurements suggest the presence of oligomeric species in BRMS1 $1_{51-84}$ and BRMS1 $1_{51-98} 14$. We wondered whether both proteins behaved as oligomers in solution by using a battery of spectroscopic techniques, namely fluorescence, CD and NMR.

NMR: The $1 \mathrm{D}-{ }^{1} \mathrm{H}-\mathrm{NMR}$ spectra of both proteins shows most of the amide protons clustered between 8.0 and 8.5 ppm (Fig. 5 A), close to the expected values for random-coil chains $^{19}$. Furthermore, their methyl groups are clustered between 0.8 and 1.0 ppm (Fig. 5 B); both $\mathrm{BRMS} 1_{51-84}$ and BRMS1 $1_{51-98}$ showed several shoulders at the main peak around 0.70 ppm, which suggest local structure around the methyl groups of a particular valine, leucine or isoleucine probably close to the sole aromatic ring in both proteins (Tyr53). 
Therefore, the NMR spectra suggest that in aqueous solution BRMS1 $1_{51-84}$ is mainly a disordered protein, with evidence of residual structure (as judged by the up-field shifted methyl protons). However, it is possible that as shown by the X-ray structure, the structure of BRMS1 $1_{51-84}$ and that of BRMS1 $1_{51-98}$, in aqueous solution was mainly helical, since helical folds show a tendency to have all the amide protons clustered in the nearby region expected for random-coil. Thus, the observed 1D-NMR spectra (Fig. 5) would correspond to those of highly helical folds, and therefore, we decided to find out whether those helical structures were rigid, and if they existed, how they changed with temperature, $\mathrm{pH}$ or chemical denaturants. To address these questions we carried out biophysical studies by using other spectroscopic techniques.

Fluorescence: We have used fluorescence spectroscopy to map any change in the tertiary structure of both proteins ${ }^{20}$. Since BRMS1 $1_{51-84}$ and BRMS1 $1_{51-98}$ have no tryptophan residues, and they contain a sole tyrosine (Tyr53, at the $\mathrm{N}$ terminus of both protein species), the fluorescence spectra of BRMS1 $1_{51-84}$ and $B R M S 1_{51-98}$ had a maximum wavelength at 306 nm, which was not modified except at basic pHs (Fig. 6 A, red circles); this titration probably corresponds to that of tyrosine residues ${ }^{21}$. The basic denaturation was also observed in BRMS1 $1_{51-98}$ (Fig. 6 A, blue squares), but in addition, the fluorescence intensities of BRMS1 $1_{51-}$ 98 changed at around $\mathrm{pH} 4$, decreasing at any of the explored wavelengths. The fitting of the titration curve at acidic $\mathrm{pH}$ yielded a $\mathrm{p} K_{\mathrm{a}}=4.6 \pm 0.3$; this value is close to that expected for glutamic or aspartic residues ${ }^{21}$.

ANS is used as a fluorescence probe, which binds to spatially close solvent-exposed hydrophobic patches ${ }^{22}$, shifting the fluorescence maxima from 520 (isolated ANS) to $480 \mathrm{~nm}$ (ANS-bound to a polypeptide hydrophobic patch). At low pHs, the ANS fluorescence intensity at $480 \mathrm{~nm}$ was large in the presence of BRMS1 $1_{51-98}$ (Fig. $6 \mathrm{~B}$, blue squares), indicating solvent-exposure of hydrophobic patches; as the $\mathrm{pH}$ was increased, the intensity at $480 \mathrm{~nm}$ decreased, and then, burial of solvent-exposed hydrophobic patches occurs (Fig. 6 B). The $\mathrm{p} K_{\mathrm{a}}$ corresponding to this acidic transition was $4.5 \pm 0.3$. Conversely, the ANS emission 
spectrum of BRMS1 $1_{51-84}$ (Fig. $6 \mathrm{~B}$, red circles) showed a small titration with a $\mathrm{p} K_{\mathrm{a}}=5.6 \pm 0.3$. Both $\mathrm{p} K_{\mathrm{a}}$ values are close to those expected for glutamic or aspartic residues ${ }^{21}$.

The thermal denaturations of BRMS1 $1_{51-98}$ and $B R M S 1_{51-84}$ showed a linear decrease in the fluorescence emission intensity at any of the explored wavelengths, as the temperature was increased, at any pH (Fig. 6 C). These data suggest that the structure in both BRMS1s does not unfold cooperatively (that would result in a sigmoidal-like curve).

Circular dichroism: We used far-UV CD in the analysis of the structure and conformational stability of $\mathrm{BRMS} 1_{51-98}$ and $\mathrm{BRMS} 1_{51-84}$ as a spectroscopic probe that is sensitive to secondary structure ${ }^{23 ; 24}$. The $\mathrm{CD}$ spectrum, at $298 \mathrm{~K}$ and physiological $\mathrm{pH}$, of both BRMS1 species showed the typical shape of $\alpha$-helix proteins with two minima around 208 and $222 \mathrm{~nm}^{23 ; 24}$ (Fig. 7 A), in agreement with the X-ray results. Furthermore, the molar ellipticity at $222 \mathrm{~nm}$ for BRMS1 $1_{51-98}$ showed a larger absolute value (Fig. $7 \mathrm{~A}$, blue squares) than that of BRMS1 $1_{51-84}$ (Fig. $7 \mathrm{~A}$, red circles). The ellipticity at $222 \mathrm{~nm}$, as the $\mathrm{pH}$ was varied, did not show any titration in $\mathrm{BRMS1}_{51-84}$, except at basic pHs (Fig. $7 \mathrm{~B}$, red circles). However, BRMS ${ }_{51-98}$ showed, in addition, another transition with $\mathrm{p} K_{\mathrm{a}}=4.1 \pm 0.2$ (Fig. $7 \mathrm{~B}$, blue squares). This behaviour in the titration of $\mathrm{BRMS} 1_{51-98}$ is similar to that observed by fluorescence (see above). On the other hand, the basic titration in both species could monitor the titration of Tyr53, which parallels the changes in secondary structure, monitored by CD.

Thermal denaturations at several pHs (Fig. 7 C) did not show a sigmoidal-like behaviour. Moreover, the GdmCl-induced chemical denaturations, showed a non-sigmoidal decrease in the absolute value as the [GdmCl] was raised (Fig. $7 \mathrm{D}$ ). Thus, there is evidence of secondary structure (probably helical) in both species, as judged by the shape of the CD, but this structure is flickering and not stable.

If there is helical structure which is not stable enough, could this helical structure be stabilized by TFE addition, as it occurs in other short peptides? Trying to address this question, we carried out TFE titrations followed by CD (Fig. 1 SM). The TFE titrations for both BRMS1s did not follow a two-state sigmoidal behaviour (as suggested by the absence of 
an isodichroic wavelength); but, the ellipticity at $222 \mathrm{~nm}$ decreased monotonically as the concentration of TFE was raised, suggesting the stabilization of the already present helical structure. We think that since an increase in helical length it is unlikely, the increase in ellipticity might be attributed to helical stabilization. Finally, the 1D-NMR experiments in 80 $\%$ TFE, with the shorter BRMS1 $1_{51-84}$ showed broad signals in the amide region, clustered between 8.0 and 8.5 ppm (Fig. $2 \mathrm{SM}$, top), and the methyl region did not show a large signal dispersion (Fig. 2 SM, bottom).

\section{DISCUSSION}

Comparison between BRMS1 $1_{51-84}$ and BRMS1 $1_{51-98}$ structures

Both BRMS1 $1_{51-84}$ and BRMS1 $1_{51-98}$ crystal structures show the overall architecture of antiparallel coiled coils with a clear conservation of the aminoacid positions that define the heptade repeats. However, the overall bending of the coiled coils appears to be slightly different (Fig. 8). Backbone atoms superposition between individual A chains of BRMS1 $1_{51-84}$ and $\mathrm{BRMS}_{51-98}$ resulted in and RMSD of $1.18 \AA$. Corresponding partner $\mathrm{B}$ chains superposed with an RMSD of $1.28 \AA$.

Residues $E^{51}$ DYERRSEC $^{60}$ do not contribute to the $\mathrm{BRMS1}_{51-84}$ antiparallel coiledcoil but they do so in BRMS1 $_{51-98}$, which contain the additional sequence $\mathrm{Q}^{85}$ LRLRLEEVGAERA $^{98}$. It is possible that the larger changes observed in BRMS1 $1_{51-98}$ in the ANS experiments (and then, in the burial of hydrophobic surface, when the protein folds from acidic $\mathrm{pHs}$ ) could be due to the acquisition of a more rigid helix-like structure of the $\mathrm{N}$ terminus of $\mathrm{BRM} 1_{51-98}$, together with the presence of the additional tail at its $\mathrm{C}$ terminus, where several Glu residues are also present.

BRMS1 $1_{51-84}$ crystal structure shows an antiparallel coiled coil, an observation compatible with size exclusion chromatography ${ }^{17}$ and AUC data (Fig. 4), which suggest the formation of the coiled-coil motif with a molecular weight of $9.9 \mathrm{kDa}$. In turn, AUC experiments suggest that $\mathrm{BRMS} 1_{51-98}$ is in a hexameric state, compatible with the trimer of 
antiparallel coiled coils seen in its crystal structure ${ }^{14}$. Therefore, residues Q85-G94, as they appear in the structure, seem to be important for oligomerisation although they do not establish direct contacts in the hexameric formation. A possible explanation would be that residues Q85-G94 stabilise the conformation of residues E51-S58 by interacting with them in the context of the antiparallel coiled coil. The latter residues are indeed directly involved in the trimerisation of the coiled coils. The lack of stabilisation of E51-S58 also affects the conformation of the N-terminal ends in $\mathrm{BRMS} 1_{51-84}$ which are located further apart than in BRMS1 $1_{51-98}$ hexamer, preventing contacts between important residues (as is the case of R56 and R57) that mediate the N-termini interaction and consequent assembly of the hexamer (Fig. $3 \mathrm{SM})^{14}$.

How can BRMS1 $1_{51-84}$ and BRMS1 $1_{51-98}$ behave as proteins with non-rigid structure in solution?

Several observations lead to the conclusion that BRMS1 N-terminal coiled-coil has the ability to adopt at least a partially ordered structure. We have shown first in this work, and previously reported ${ }^{14}$, that $\mathrm{BRMS} 1_{51-84}$ and $\mathrm{BRMS} 1_{51-98}$, respectively adopt a coiled-coil structure in the crystals. Moreover, the far-UV CD spectra of both fragments also indicate the presence of a helical structure. In addition, apart from the crystal structures, we have also detected a higher degree of organisation in solution since AUC and DOSY measurements suggest that both BRMS1 constructs form oligomers. However, mono-dimensional NMR (since the 1D- spectrum could also resemble that of a helical conformation with few aromatic side chains), together with thermal and chemical denaturation assays are suggesting that both BRMS1 constructs behave as non-rigid structures or as disordered proteins. This apparent contradiction can be explained by the different explored properties of each technique. AUC and DOSY are sensitive to distances and/or to molecular size, and it should be emphasized that distances in a highly dynamic system should be interpreted in terms of distance distributions (from the average population) rather than unique distances. Thus, it could be that DOSY and AUC would be reporting a highly dynamic equilibrium in aqueous solution where 
the protein partially unfolds (and hence the observed 1D-NMR spectrum), while keeping a helical and oligomeric state, where the partially folded monomeric species self-associate (and so, the AUC and DOSY results). The far-UV CD would report the presence of a helical structure, whereas the NMR spectra, due to its different spectroscopic time scale, would probably report on the weighted average of several conformational states and/or on helical populations (self-associated or not).

The fact that both BRMS1 constructs appear to have a non-rigid structure in solution does not exclude the presence of ordered states in equilibrium. In fact, compact structures have been observed to exist in the conformational ensembles of intrinsically disordered proteins, by using experimental and theoretical approaches ${ }^{25}$. Indeed, it has been suggested that a bias toward oligomerisation in natively disordered proteins could either represent a mechanism for protection against proteolytic degradation $\left({ }^{26}\right.$, and references therein), or in order to perform their molecular functions ${ }^{27}$. The highly dynamic conformational ensembles explored by $\mathrm{BRMS} 1_{51-84}$ and $\mathrm{BRMS} 1_{51-98}$ allow for fast intrinsic fluctuations that grant the efficient sampling of the relevant conformational space to carry out their functions. Crystallisation might shift this equilibrium towards the ordered state, and in this sense lattice contacts appear to be more efficient in stabilizing an ordered conformation of an intrinsically disordered protein, than the crowded conditions in a solution buffer $^{28 ; 29}$. On the other hand, additional contacts between the helices outside the coiled-coil pairs established during the crystallisation process, could lead to a single energy minimum, underrepresented in the dynamic solution, especially when activation barriers are comparable to the thermal energy in the sample ${ }^{30}$. In any case, our results would support the recent observation that there is not always a good correlation between the probability of obtaining suitable crystals for structural studies and the quality of the NMR spectra ${ }^{31}$. Both techniques could and should be complementary since they might be covering different areas of the conformational space.

This dynamic conformational ensemble might be of relevance to the biological role of BRMS1 N-terminal coiled coil, since it involves the functional NES signal (residues 74-91 ${ }^{13}$ ). 
All the residues that define the consensus export signal, except L88 (F79, L83 and L86), appear to be occluded in the context of the antiparallel coiled coil. In fact, they constitute some of the positions that define the heptad repeat in the BRMS1 $1_{51-98}$ structure $^{14}$. These residues are even less accessible in the context of the trimer of antiparallel coiled coils (Fig. 9) which have been shown to hold stable in solution to as little as $8.35 \mu \mathrm{M}^{14}$. Therefore, the observed conformational ensemble would provide a rationale accounting for the nuclear export function of these residues: the existence of dynamic conformers might allow the exposure of the key residues and interaction of the NES with cellular transporters in order to perform its export function from the nucleus onto the cytoplasm. BRMS1 is regulated by epigenetics phenomena and its expression leads to suppression of metastasis. Most of the molecular functions reported for BRMS1, including its role as transcriptional inhibitor, require a BRMS1 nuclear localisation. It has been proposed that the nuclear-cytoplasmic shuttling could suppose another level of BRMS1 regulation and in fact, it has been reported that a BRMS1 localization shift to the cytoplasm is associated with highly proliferative ERnegative breast cancers ${ }^{15}$. A differential behaviour depending on BRMS1 localization has also been reported in melanoma ${ }^{16}$. In a sense, the oligomerisation shown by the $\mathrm{N}$-terminal coiled coil might be important to keep BRMS1 in the nucleus, where it is more likely to engage in activities resulting in transcriptional repression and metastasis suppression. The occlusion of the key residues in the NES either by the formation of the antiparallel coiled-coil or its trimerisation might then contribute to additional levels of BRMS1 regulation. Other regions at the N-terminal coiled coil might also be relevant for BRMS1 function since BRMS1 Nterminal coiled coil has been shown to be important for interacting with other cellular partners $^{12}$.

In summary, our results suggest that the oligomerisation state of BRMS1 induced by its N-terminal coiled coil could have an impact on the regulation of BRMS1, molecular functions and metastasis since antimetastatic properties of BRMS1 involve transcriptional repression and a localization shift to the cytoplasm is associated with highly proliferative 
breast cancers. Taken together, the reported dynamic behaviour would provide a rationale to make compatible the reported BRMS1 N-terminal coiled coil oligomerisation state with the nuclear export functionality or its possible interaction with other cellular partners.

\section{MATERIALS AND METHODS}

Materials: Deuterium oxide and deuterated $\mathrm{d}_{3}$-TFE (2,2,2,-trifluoroethan-1-ol) were obtained from Apollo Scientific (Stockport, UK), and the sodium trimethylsilyl $\left[2,2,3,3-{ }^{2} \mathrm{H}_{4}\right]$ propionate, TSP, was from Sigma (St. Louis, MO). Dialysis tubing, with a molecular weight cut-off of 3,500 Da (Spectrapor), was from Spectrum Laboratories (Japan). Amicon centrifugal devices with a molecular weight cut-off of 3,500 Da were from Millipore (Millipore, MA). Standard suppliers were used for all other chemicals. Water was deionized and purified on a Millipore system.

Protein expression and purification: $\mathrm{BRMS} 1_{51-84}$ and $\mathrm{BRMS} 1_{51-98}$ proteins were expressed in heterologous expression systems using E. coli strains and purified as previously described ( ${ }^{14 ;} 17$ for BRMS1 $1_{51-84}$ and BRMS1 $1_{51-98}$ respectively).

Crystallisation, data collection and structure determination: Crystals of BRMS1 $1_{51-84}$ with typical dimensions of $0.35 \mathrm{~mm} \times 0.2 \mathrm{~mm} \times 0.1 \mathrm{~mm}$ were obtained as previously reported $^{17}$, and were used to perform soaking with $2 \mathrm{mM}$ mercury(II) nitrate monohydrate (Hampton Reasearch) during 15 hours. Derivatised crystals were cryoprotected by immersion in Paratone-N (Hampton Research), mounted on cryo-loops and flashed-cooled at $130 \mathrm{~K}$ under a cryogenic nitrogen stream, in order to be irradiated under the ID23-1 beamline at ESRF (Grenoble), at a wavelength of $0.9729 \AA$ using an ADSC CCD detector. The images were collected using the software MXCuBE (MX Customised Beamline Environment). The data were processed with the programs MOSFLM v.7.0.2 for reflection, indexing and integration and SCALA v.3.3.2 for scaling and merging, both from the CCP4 program suite ${ }^{32}$; ${ }^{33}$, obtaining the final statistics shown in Table 1. 
The BRMS1 $1_{51-84}$ structure was solved by SIRAS using a native ${ }^{17}$ and the mercury derivative datasets, by means of the SHELX package ${ }^{34}$ through the hkl2map graphical userinterface ${ }^{35 ;} 36$. In order to solve the phases, data including $3.5 \AA$ of resolution were used in SHELXD and a solvent content of $60 \%$ was assumed through 20 phasing/density modification cycles in SHELXE. Once the phases were obtained, by using only the 2 heavy atom positions with more than $80 \%$ occupancy, an initial model was constructed by combining the outputs from the ARP/wARP and Buccaneer programs $\left(\mathrm{CCP} 4\right.$ package $\left.^{32 ; 33}\right)$ as well as Resolve (AutoBuild wizard from PHENIX) ${ }^{37}$. Further work was carried out using the native dataset $\left(2.0 \AA,{ }^{17}\right)$.

We obtained the final model by iterative manual model building of BRMS1 $1_{51-84}$ using the graphics program $\operatorname{Coot}^{38}$ combined with restrained refinement cycles in the reciprocal space, using Translation/Libration/Screw (TLS) refinement ${ }^{39}$ (8 TLS groups, one per chain) and Babinet scaling as implemented in REFMAC5 $5^{40}$. Initial parameters for TLS refinement were generated using the TLSMD web server ${ }^{41}$. The final confidence parameters $R$ and $R_{\text {free, }}$ the $\mathrm{B}$ factors and the RMSD of bonds and angles with respect to the ideal values used in the refinement, were checked using Sfcheck, Procheck and Baverage $\left(\mathrm{CCP}^{32}{ }^{33}\right)$. MolProbity ${ }^{42}$ was used for steric and geometric analyses and Contact from CCP4 and Monster ${ }^{43}$ programs to analyse the residue contacts. The heptad repeat assignment on BRMS1 $1_{51-84}$ molecule was conducted with the program Twister ${ }^{44}$. Superposition of molecules was analysed by the SuperPose server (Version 1.0) $)^{45}$.

BRMS1 $1_{51-84}$ coordinates and structure factors have been deposited in the Protein Data Bank with code 4AUV and R4AUVSF respectively.

Fluorescence: Spectra were collected on a Cary Eclipse spectrofluorometer (Agilent, Spain) interfaced with a Peltier system. A 1-cm-path-length quartz cell (Hellma) was used. The proper blank solutions were subtracted in all cases.

(a) Steady state fluorescence measurements- Spectra of BRMS1 $1_{51-84}$ and BRMS1 $1_{51-98}$ in aqueous solution were acquired by excitation at $280 \mathrm{~nm}$; the emission spectra were 
collected between 300 and $400 \mathrm{~nm}$. The excitation and emission slits were set to $5 \mathrm{~nm}$, and the response to $1 \mathrm{~nm}$. Protein concentration was in all cases $10 \mu \mathrm{M}$ of the protomer.

For the experiments at different pHs of both BRMS1 proteins, the buffers used were: $\mathrm{pH}$ 2.0-3.0, phosphoric acid; $\mathrm{pH}$ 3.0-4.0, formic acid; $\mathrm{pH}$ 4.0-5.5, acetic acid; $\mathrm{pH}$ 6.0-7.0, $\mathrm{NaH}_{2} \mathrm{PO}_{4} ; \mathrm{pH}$ 7.5-9.0, TRIS base; $\mathrm{pH}$ 9.5-11.0, $\mathrm{Na}_{2} \mathrm{CO}_{3}$. The $\mathrm{pH}$ was measured with an ultrathin Aldrich electrode in a Radiometer (Copenhagen) $\mathrm{pH}$-meter. The samples were prepared the day before and left overnight at $298 \mathrm{~K}$ to equilibrate. The $\mathrm{pH}$ was measured after completion of the experiments, and no significant differences were observed with those $\mathrm{pHs}$ calculated from the buffer stocks. Buffer concentration was $10 \mathrm{mM}$ in all cases. The $\mathrm{pH}-$ titration was repeated twice for each protein with new samples.

(b) Thermal denaturations- Spectra were acquired by excitation at $270 \mathrm{~nm}$; the emission fluorescence was collected at 315,335 and $350 \mathrm{~nm}$. The excitation and emission slits were set to $5 \mathrm{~nm}$; the response was $1 \mathrm{~nm}$, and the scan rate was $60 \mathrm{~K} / \mathrm{h}$, with an average data point of $1 \mathrm{~s}$. Every experiment was repeated twice with new samples.

(c) ANS titrations- Spectra were acquired with $100 \mu \mathrm{M}$ of ANS and $10 \mu \mathrm{M}$ of the corresponding BRMS1 protein in the buffers described above at $10 \mathrm{mM}$ of the final concentration; the temperature was $298 \mathrm{~K}$. Dye concentrations were determined using an extinction coefficient of $8000 \mathrm{M}^{-1} \mathrm{~cm}^{-1}$ at $370 \mathrm{~nm}$. Blank corrections were used in all cases. Experiments were acquired by excitation at $370 \mathrm{~nm}$; the emission was collected between 400 and $600 \mathrm{~nm}$. The excitation and emission slits were set to $5 \mathrm{~nm}$, and the response was $1 \mathrm{~nm}$. Buffer concentration was $10 \mathrm{mM}$ in all cases.

Circular dichroism: Spectra were collected on a Jasco J810 (Japan) spectropolarimeter connected to a Peltier unit. The instrument was periodically calibrated with (+)-10camphorsulfonic acid. Spectra were acquired at $298 \mathrm{~K}$ in phosphate buffer at $\mathrm{pH} 7.0(10 \mathrm{mM})$. For each experiment, corresponding blank solutions were subtracted.

(a) Steady state measurements- Spectra of both BRMS1s in the far-UV CD were acquired with a response time of $2 \mathrm{~s}$, and averaged over 4 scans, with a scan speed of 50 
$\mathrm{nm} / \mathrm{min}$. The step resolution was $0.2 \mathrm{~nm}$, and the band-width was $1 \mathrm{~nm}$. Molar ellipticity was obtained as described $^{24}$. The concentration of each BRMS1 protein was $10 \mu \mathrm{M}$ of protomer. The cell path-length was $0.1 \mathrm{~cm}$. Every experiment was repeated three times with new samples.

For the experiments at different pHs, buffers and protocols were the same as described above for fluorescence assays. The $\mathrm{pH}$-titration was repeated twice with new samples. Fitting of the resulting sigmoidal $\mathrm{pH}$-titration curves to the Henderson-Hasselbalch equation was carried out by using Kaleidagraph (Abelbeck software).

In the GdmCl-denaturations, far-UV CD spectra were acquired at a scan speed of 50 $\mathrm{nm} / \mathrm{min}$, and 6 scans were recorded and averaged at $298 \mathrm{~K}$. The response time was $2 \mathrm{sec}$. The cell path-length was $0.1 \mathrm{~cm}$, with a protein concentration of $10 \mu \mathrm{M}$ for both $\mathrm{BRMS1}_{51-98}$ and $\mathrm{BRMS1}_{51-84}$. The corresponding $[\mathrm{GdmCl}]$ was prepared from a stock solution at $7 \mathrm{M}$. The samples were prepared the day before and left overnight at $298 \mathrm{~K}$ to equilibrate. The chemical denaturations were fully reversible, and they were repeated three times with new samples.

In the TFE titrations, solutions ranging from 0 to $80 \%$ of TFE (volume/volume) were prepared with protein concentrations of 10 and $15 \mu \mathrm{M}$ for both $\mathrm{BRMS1} 1_{51-84}$ and $\mathrm{BRMS} 1_{51-98}$, respectively. The far-UV CD spectra were acquired at a scan speed of $50 \mathrm{~nm} / \mathrm{min}$, and 6 scans were recorded and averaged at $298 \mathrm{~K}$. The response time was $2 \mathrm{sec}$, and the cell path-length was $0.1 \mathrm{~cm}$.

(b) Thermal-denaturations- Thermal-denaturations were carried out by following the changes at $222 \mathrm{~nm}$, at $60 \mathrm{~K} / \mathrm{h}$, with a response time of $8 \mathrm{~s}$. All the denaturations were reversible. The possibility of drifting of the $\mathrm{CD}$ spectropolarimeter was tested by running two samples containing only buffer, before and after the thermal experiments. No difference was observed between the scans. The experiments were repeated twice with new samples. Fitting of the sigmoidal curves was carried out as described ${ }^{46}$, by using Kaleidagraph (Abelbeck software). 
NMR spectroscopy: The NMR experiments in aqueous solution and in $40 \%$ TFE were acquired at $293 \mathrm{~K}$ on a Bruker Avance DRX-500 spectrometer (Bruker GmbH, Germany), equipped with a triple resonance probe and z-pulse field gradients. Processing of spectra was carried out with the XWINNMR software. All experiments were carried out at pH 7.0, $10 \mathrm{mM}$ phosphate buffer, and with a protein concentration of $100 \mu \mathrm{M}$. Samples were concentrated by using Amicon centrifugal devices.

(a) $1 D-{ }^{1} H$-NMR experiments- TSP was used as the internal chemical shift reference in the $1 \mathrm{D}-{ }^{1} \mathrm{H}-\mathrm{NMR}$ spectra of both BRMS1 ${ }_{51-84}$ and $\mathrm{BRMS} 1_{51-98}$. Water was suppressed with the WATERGATE sequence $^{47}$. Usually, 512 scans were acquired with a spectral width of $12 \mathrm{ppm}$, with $16 \mathrm{~K}$ data points in the time domain. The data matrix was zero filled to $32 \mathrm{~K}$ during processing. Experiments in aqueous solution were acquired with both proteins, and, we also acquired a spectrum in $40 \%$ TFE with BRMS151-84.

(b) Translational diffusion NMR experiments (DOSY)- Translational self-diffusion measurements were performed with the pulsed-gradient spin-echo sequence for BRMS1 $1_{51-84}$. The following relationship exists between the translational self-diffusion constant, $D$, and the delays during acquisition ${ }^{46}: \frac{I}{I_{0}}=-\exp \left(D \gamma_{H}^{2} \delta^{2} G^{2}\left(\Delta-\frac{\delta}{3}-\frac{\tau}{2}\right)\right)$, where $I$ is the measured peak intensity of a particular (or a group of) resonance(s); $I_{0}$ is the maximum peak intensity of the same resonance(s) at the smaller gradient strength; $D$ is the translational self-diffusion constant (in $\mathrm{cm}^{2} \mathrm{~s}^{-1}$ ); $\delta$ is the duration (in s) of the gradient; $G$ is the gradient strength (in $\mathrm{T}$ $\mathrm{cm}^{-1}$ ); $\Delta$ is the time (in s) between the gradients; $\gamma_{\mathrm{H}}$ is the gyromagnetic constant of the proton; and $\tau$ is the recovery delay between the bipolar gradients (100 $\mu$ s in our experiments). Data are plotted as $I / I_{0}$ versus $G^{2}$, and the exponential factor of the resulting curve is $D \gamma_{H}^{2} \delta^{2}\left(\Delta-\frac{\delta}{3}-\frac{\tau}{2}\right)$, from where $D$ can be easily obtained. The duration of the gradient was $1.9 \mathrm{~ms}$, and the time between both gradients was $125 \mathrm{~ms}$. The methyl groups between 0.8 and $1 \mathrm{ppm}$ were used for integration. The gradient strength was calibrated by using the value of $D$ 
for the residual proton water line in a sample containing $100 \% \mathrm{D}_{2} \mathrm{O}$ in a $5-\mathrm{mm}$ tube ${ }^{46}$. The hydrodynamic radius, $R_{h}$, was obtained by assuming that the $R_{h}$ of dioxane is $2.12 \AA^{18}$. The DOSY measurement was repeated twice. It is important to indicate at this stage, that the determined $D$ is the weighted average of all the translational diffusion coefficients of all species present in solution.

Analytical ultracentrifugation: The sample protein at $0.5 \mathrm{mg} / \mathrm{mL}(114.5 \mu \mathrm{M})$ in buffer containing $20 \mathrm{mM}$ TRIS- $\mathrm{HCl} \mathrm{pH} 7.5,50 \mathrm{mM} \mathrm{NaCl}$ and $1 \mathrm{mM}$ DTT was pre-centrifuged at $16,000 \mathrm{x} g$ before ultracentrifugation protocol. The experiments were conducted at $293 \mathrm{~K}$.

(a) Velocity measurements: Sedimentation velocity experiment was performed on an Optima XL-I (Beckman, CA), using an AnTi50 rotor and a standard double-sector Eponcharcoal center pieces (1.2 cm optical path length). $400 \mu \mathrm{L}$ of sample and reference solution were loaded and sedimented at 48,000 rpm during 3 hours and 30 minutes, registering successive entries every minute. The evolution of the resulting concentration gradient of material in the cell as a function of time and radial position was monitored by absorbance at the wavelength $230 \mathrm{~nm}$. Differential sedimentation coefficient distributions (c(s)) were calculated by least-squares boundary modelling of sedimentation velocity data using SedFit software (Version 12.52) as described ${ }^{48}$. The calculated frictional ratios were used to transform the $\mathrm{c}(\mathrm{s})$ distribution into the corresponding molar mass distribution ${ }^{49 ; 50}$.

(b) Sedimentation Equilibrium: An Optima XL-I (Beckman, CA) analytical ultracentrifuge equipped with UV-visible absorbance optics was employed for analytical ultracentrifugation measurements by using an An50Ti rotor. Short column $(85 \mu \mathrm{L})$ sedimentation equilibrium runs were carried out at multiple speeds $(30,36$, and $48 \mathrm{krpm})$ by taking absorbance scans at the wavelength $235 \mathrm{~nm}$. After the equilibrium scans, a high-speed centrifugation run at $45 \mathrm{krpm}$ was done to estimate the corresponding baseline offsets. The weight-average buoyant molecular weight of $B R M S 1_{51-84}$ was calculated using the HeteroAnalysis program (Version 1.1.44) ${ }^{51}$. The molecular weight of the protein was determined 
from the experimental buoyant masses using the values of $0.7300 \mathrm{~cm}^{3} / \mathrm{g}$ and $1.0009 \mathrm{~g} / \mathrm{cm}^{3}$ as the partial specific volume and density, respectively.

\section{ACKNOWLEDGMENTS:}

Crystallographic data collection experiments were performed on the ID14-1 and ID23-1 beamlines at the European Synchrotron Radiation Facility (ESRF), Grenoble, France. We are grateful to local contact at ESRF for providing assistance in using both beamlines.

Work at JLN laboratory was supported by the Spanish Ministerio de Ciencia e Innovación (MCINN) (CTQ2011-24393, CSD2008-00005), and intramural BIFI 2011 grant. Work at JB laboratory was supported by Ministerio de Ciencia e Innovación (SAF200804048-E, SAF2009-10667), Conselleria de Sanitat, Generalitat Valenciana AP-001/10, CSIC (200820I020) and Fundación Mutua Madrileña, Spain.

The authors thank Carlos Alfonso Botello (Servicio de Ultracentrifugación Analítica, Centro de Investigaciones Biológicas, CSIC) for analytical ultracentrifugation.

\section{REFERENCES}

1. Hurst, D. R. \& Welch, D. R. (2011). Metastasis suppressor genes at the interface between the environment and tumor cell growth. Int Rev Cell Mol Biol 286, 107-80.

2. Metge, B. J., Frost, A. R., King, J. A., Dyess, D. L., Welch, D. R., Samant, R. S. \& Shevde, L. A. (2008). Epigenetic silencing contributes to the loss of BRMS1 expression in breast cancer. Clin Exp Metastasis 25, 753-63.

3. Cicek, M., Fukuyama, R., Cicek, M. S., Sizemore, S., Welch, D. R., Sizemore, N. \& Casey, G. (2009). BRMS1 contributes to the negative regulation of uPA gene expression through recruitment of HDAC1 to the NF-kappaB binding site of the uPA promoter. Clin Exp Metastasis 26, 229-37.

4. Hurst, D. R., Xie, Y., Vaidya, K. S., Mehta, A., Moore, B. P., Accavitti-Loper, M. A., Samant, R. S., Saxena, R., Silveira, A. C. \& Welch, D. R. (2008). Alterations of 
BRMS1-ARID4A interaction modify gene expression but still suppress metastasis in human breast cancer cells. J Biol Chem 283, 7438-44.

5. Rivera, J., Megias, D. \& Bravo, J. (2007). Proteomics-based strategy to delineate the molecular mechanisms of the metastasis suppressor gene BRMS1. J Proteome Res 6 , 4006-18.

6. Samant, R. S., Clark, D. W., Fillmore, R. A., Cicek, M., Metge, B. J., Chandramouli, K. H., Chambers, A. F., Casey, G., Welch, D. R. \& Shevde, L. A. (2007). Breast cancer metastasis suppressor 1 (BRMS1) inhibits osteopontin transcription by abrogating NFkappaB activation. Mol Cancer 6, 6 .

7. Yang, J., Zhang, B., Lin, Y., Yang, Y., Liu, X. \& Lu, F. (2008). Breast cancer metastasis suppressor 1 inhibits SDF-1alpha-induced migration of non-small cell lung cancer by decreasing CXCR4 expression. Cancer Lett 269, 46-56.

8. Doyon, Y., Cayrou, C., Ullah, M., Landry, A. J., Cote, V., Selleck, W., Lane, W. S., Tan, S., Yang, X. J. \& Cote, J. (2006). ING tumor suppressor proteins are critical regulators of chromatin acetylation required for genome expression and perpetuation. Mol Cell 21, 51-64.

9. Meehan, W. J., Samant, R. S., Hopper, J. E., Carrozza, M. J., Shevde, L. A., Workman, J. L., Eckert, K. A., Verderame, M. F. \& Welch, D. R. (2004). Breast cancer metastasis suppressor 1 (BRMS1) forms complexes with retinoblastoma-binding protein 1 (RBP1) and the mSin3 histone deacetylase complex and represses transcription. J Biol Chem 279, 1562-9.

10. Liu, Y., Smith, P. W. \& Jones, D. R. (2006). Breast cancer metastasis suppressor 1 functions as a corepressor by enhancing histone deacetylase 1-mediated deacetylation of RelA/p65 and promoting apoptosis. Mol Cell Biol 26, 8683-96.

11. Hurst, D. R., Mehta, A., Moore, B. P., Phadke, P. A., Meehan, W. J., Accavitti, M. A., Shevde, L. A., Hopper, J. E., Xie, Y., Welch, D. R. \& Samant, R. S. (2006). Breast 
cancer metastasis suppressor 1 (BRMS1) is stabilized by the Hsp90 chaperone. Biochem Biophys Res Commun 348, 1429-35.

12. Rivera, J., Megias, D. \& Bravo, J. (2010). Sorting nexin 6 interacts with breast cancer metastasis suppressor-1 and promotes transcriptional repression. J Cell Biochem 111, 1464-72.

13. Rivera, J., Megias, D., Navas, C. \& Bravo, J. (2009). Identification of essential sequences for cellular localization in BRMS1 metastasis suppressor. PLoS One 4, e6433.

14. Spinola-Amilibia, M., Rivera, J., Ortiz-Lombardia, M., Romero, A., Neira, J. L. \& Bravo, J. (2011). The structure of BRMS1 nuclear export signal and SNX6 interacting region reveals a hexamer formed by antiparallel coiled coils. J Mol Biol 411, 1114-27.

15. Frolova, N., Edmonds, M. D., Bodenstine, T. M., Seitz, R., Johnson, M. R., Feng, R., Welch, D. R. \& Frost, A. R. (2009). A shift from nuclear to cytoplasmic breast cancer metastasis suppressor 1 expression is associated with highly proliferative estrogen receptor-negative breast cancers. Tumour Biol 30, 148-59.

16. Slipicevic, A., Holm, R., Emilsen, E., Ree Rosnes, A. K., Welch, D. R., Maelandsmo, G. M. \& Florenes, V. A. (2012). Cytoplasmic BRMS1 expression in malignant melanoma is associated with increased disease-free survival. BMC Cancer 12, 73.

17. Spinola-Amilibia, M., Rivera, J. \& Bravo, J. (2008). Crystallization and preliminary X-ray diffraction analysis of a breast cancer metastasis suppressor 1 predicted coiledcoil region. Acta Crystallogr Sect F Struct Biol Cryst Commun 64, 1156-8.

18. Wilkins, D. K., Grimshaw, S. B., Receveur, V., Dobson, C. M., Jones, J. A. \& Smith, L. J. (1999). Hydrodynamic radii of native and denatured proteins measured by pulse field gradient NMR techniques. Biochemistry 38, 16424-31.

19. Wüthrich, K., Ed. (1986). NMR of proteins and nucleic acids. New York: Wiley. 
20. Schmid, F. X., Ed. (1997). Optical spectroscopy to characterize protein conformation and conformational changes. Vol. 11. Protein structure: a practical approach. Edited by Creighton, T. E. New York: Oxford University Press.

21. Thurlkill, R. L., Grimsley, G. R., Scholtz, J. M. \& Pace, C. N. (2006). pK values of the ionizable groups of proteins. Protein Sci 15, 1214-8.

22. Semisotnov, G. V., Rodionova, N. A., Razgulyaev, O. I., Uversky, V. N., Gripas, A. F. \& Gilmanshin, R. I. (1991). Study of the "molten globule" intermediate state in protein folding by a hydrophobic fluorescent probe. Biopolymers 31, 119-28.

23. Kelly, S. M. \& Price, N. C. (2000). The use of circular dichroism in the investigation of protein structure and function. Curr Protein Pept Sci 1, 349-84.

24. Woody, R. W. (1995). Circular dichroism. Methods Enzymol 246, 34-71.

25. Szasz, C. S., Alexa, A., Toth, K., Rakacs, M., Langowski, J. \& Tompa, P. (2011). Protein disorder prevails under crowded conditions. Biochemistry 50, 5834-44.

26. Konrat, R. (2010). The meandering of disordered proteins in conformational space. Structure 18, 416-9.

27. Danielsson, J., Liljedahl, L., Barany-Wallje, E., Sonderby, P., Kristensen, L. H., Martinez-Yamout, M. A., Dyson, H. J., Wright, P. E., Poulsen, F. M., Maler, L., Graslund, A. \& Kragelund, B. B. (2008). The intrinsically disordered RNR inhibitor Sml1 is a dynamic dimer. Biochemistry 47, 13428-37.

28. Kingston, R. L., Gay, L. S., Baase, W. S. \& Matthews, B. W. (2008). Structure of the nucleocapsid-binding domain from the mumps virus polymerase; an example of protein folding induced by crystallization. J Mol Biol 379, 719-31.

29. Sigalov, A. B. (2010). Protein intrinsic disorder and oligomericity in cell signaling. Mol Biosyst 6, 451-61.

30. Kwan, A. H., Mobli, M., Gooley, P. R., King, G. F. \& Mackay, J. P. (2011). Macromolecular NMR spectroscopy for the non-spectroscopist. FEBS J 278, 687-703. 
31. Snyder, D. A., Chen, Y., Denissova, N. G., Acton, T., Aramini, J. M., Ciano, M., Karlin, R., Liu, J., Manor, P., Rajan, P. A., Rossi, P., Swapna, G. V., Xiao, R., Rost, B., Hunt, J. \& Montelione, G. T. (2005). Comparisons of NMR spectral quality and success in crystallization demonstrate that NMR and X-ray crystallography are complementary methods for small protein structure determination. J Am Chem Soc 127, 16505-11.

32. (1994). The CCP4 suite: programs for protein crystallography. Acta Crystallogr D Biol Crystallogr 50, 760-3.

33. Winn, M. D., Ballard, C. C., Cowtan, K. D., Dodson, E. J., Emsley, P., Evans, P. R., Keegan, R. M., Krissinel, E. B., Leslie, A. G., McCoy, A., McNicholas, S. J., Murshudov, G. N., Pannu, N. S., Potterton, E. A., Powell, H. R., Read, R. J., Vagin, A. \& Wilson, K. S. (2011). Overview of the CCP4 suite and current developments. Acta Crystallogr D Biol Crystallogr 67, 235-42.

34. Sheldrick, G. M. (2010). Experimental phasing with SHELXC/D/E: combining chain tracing with density modification. Acta Crystallogr D Biol Crystallogr 66, 479-85.

35. Pape, T. \& Schneider, T. R. (2004). Hkl2map: a graphical user interface for phasing with shelx programs. Journal of Applied Crystallography 37, 843-844.

36. Sheldrick, G. M. (2008). A short history of SHELX. Acta Crystallogr A 64, 112-22.

37. Adams, P. D., Afonine, P. V., Bunkoczi, G., Chen, V. B., Davis, I. W., Echols, N., Headd, J. J., Hung, L. W., Kapral, G. J., Grosse-Kunstleve, R. W., McCoy, A. J., Moriarty, N. W., Oeffner, R., Read, R. J., Richardson, D. C., Richardson, J. S., Terwilliger, T. C. \& Zwart, P. H. (2010). PHENIX: a comprehensive Python-based system for macromolecular structure solution. Acta Crystallogr D Biol Crystallogr 66, 213-21.

38. Emsley, P., Lohkamp, B., Scott, W. G. \& Cowtan, K. (2010). Features and development of Coot. Acta Crystallogr D Biol Crystallogr 66, 486-501. 
39. Winn, M. D., Isupov, M. N. \& Murshudov, G. N. (2001). Use of TLS parameters to model anisotropic displacements in macromolecular refinement. Acta Crystallogr D Biol Crystallogr 57, 122-33.

40. Murshudov, G. N., Vagin, A. A. \& Dodson, E. J. (1997). Refinement of macromolecular structures by the maximum-likelihood method. Acta Crystallogr D Biol Crystallogr 53, 240-55.

41. Painter, J. \& Merritt, E. A. (2006). Optimal description of a protein structure in terms of multiple groups undergoing TLS motion. Acta Crystallogr D Biol Crystallogr 62 , 439-50.

42. Davis, I. W., Leaver-Fay, A., Chen, V. B., Block, J. N., Kapral, G. J., Wang, X., Murray, L. W., Arendall, W. B., 3rd, Snoeyink, J., Richardson, J. S. \& Richardson, D. C. (2007). MolProbity: all-atom contacts and structure validation for proteins and nucleic acids. Nucleic Acids Res 35, W375-83.

43. Salerno, W. J., Seaver, S. M., Armstrong, B. R. \& Radhakrishnan, I. (2004). MONSTER: inferring non-covalent interactions in macromolecular structures from atomic coordinate data. Nucleic Acids Res 32, W566-8.

44. Strelkov, S. V. \& Burkhard, P. (2002). Analysis of alpha-helical coiled coils with the program TWISTER reveals a structural mechanism for stutter compensation. J Struct Biol 137, 54-64.

45. Maiti, R., Van Domselaar, G. H., Zhang, H. \& Wishart, D. S. (2004). SuperPose: a simple server for sophisticated structural superposition. Nucleic Acids Res 32, W5904.

46. Czypionka, A., de los Panos, O. R., Mateu, M. G., Barrera, F. N., Hurtado-Gomez, E., Gomez, J., Vidal, M. \& Neira, J. L. (2007). The isolated C-terminal domain of Ring1B is a dimer made of stable, well-structured monomers. Biochemistry 46, 12764-76.

47. Piotto, M., Saudek, V. \& Sklenar, V. (1992). Gradient-tailored excitation for singlequantum NMR spectroscopy of aqueous solutions. J Biomol NMR 2, 661-5. 
48. Schuck, P. \& Rossmanith, P. (2000). Determination of the sedimentation coefficient distribution by least-squares boundary modeling. Biopolymers 54, 328-41.

49. Schuck, P., Perugini, M. A., Gonzales, N. R., Howlett, G. J. \& Schubert, D. (2002). Size-distribution analysis of proteins by analytical ultracentrifugation: strategies and application to model systems. Biophys $J$ 82, 1096-111.

50. Schuck, P. (2000). Size-distribution analysis of macromolecules by sedimentation velocity ultracentrifugation and lamm equation modeling. Biophys $J$ 78, 1606-19.

51. Cole, J. L. (2004). Analysis of heterogeneous interactions. Methods Enzymol 384, 21232.

\section{FIGURE LEGENDS}

Fig. 1: Asymmetric unit content of BRMS1 ${ }_{51-84}$ crystals. (A) Asymmetric unit content of BRMS1 $1_{51-84}$ crystals containing 8 molecules $(\mathrm{A}-\mathrm{H})$. Pairs of chains bound by a $2 \AA$ long disulphide bond (grey spheres) are shown with the same color. In the boxed panel is shown in detail the disulphide bond established between the cysteine residues 60 of chains $\mathrm{C}(C)$ and $\mathrm{H}$ $(H)$ contained in the asymmetric unit of the BRMS1 $1_{51-84}$ crystal. The electron density map $2 \mathrm{~F}_{\mathrm{o}}$

- $F_{c}$ is contoured at $1.5 \sigma$. (B) The two coiled-coil motifs established between chains A and G of the same asymmetric unit and chains A (Asym2) and C (Csym5) from symmetry-related molecules in the crystal, respectively. The symmetry-related molecules are shown in yellow.

Fig. 2: BRMS1 $1_{51-84}$ coiled coil structure. (A) Interactions between residues located at positions $a$ and $d$ in $A$-Asym 2 pair, analyzed using the Contact program (CCP4 package $\left.{ }^{32 ; 33}\right)$. The hydrophobic contacts, located at the ends of the $\alpha$ helices, between residues V61-L65L83-F79, are highlighted in green, while the polar interactions situated at the center of the double helix are shown with a red circle. (B) Contacting hydrophobicity surface between the chains $A$-Asym2 of the BRM1 $1_{51-84}$ structure, through hydrophobic residues located at positions 
$a$ and $d$ of the heptads, keeping the typical pattern of coiled-coil motifs. The chains are identified in their amino-terminals.

Fig. 3: Heptad pattern repetition in $\mathbf{B R M S 1}_{51-84}$ molecule. (A) Heptad repeat assignment on BRMS1 $1_{51-84}$ sequence. Protein sequence and the assignment of the $a$ - $g$ pattern are shown in the first and second row respectively. The heptad are delimited with boxes with different colors, and the numerical correspondence of residues in the protein sequence is shown. An asterisk is located where there is an stutter or discontinuity in the pattern. The program Twister was used for the analysis. (B) View through the longitudinal axis of the double helix A-Asym2 of BRMS1 $1_{51-84}$ structure. Localization of the residues with their correspondence in the heptad (from $a$ to $g$ ) are shown. Amino acids with the same assignment are located in the same area, and are therefore identified with a single letter.

Fig. 4: AUC of BRMS1 ${ }_{51-84}$ coiled-coil motif. The experiments were performed at $293 \mathrm{~K}$, at $114.5 \mu \mathrm{M}(0.5 \mathrm{mg} / \mathrm{mL})$ of protein concentration and in $20 \mathrm{mM}$ TRIS-HCl $\mathrm{pH} 7.5,50 \mathrm{mM}$ $\mathrm{NaCl}$ and $1 \mathrm{mM}$ DTT. (A) Sedimentation velocity data for BRMS1 $1_{51-84}$ was monitored by absorbance at the $230 \mathrm{~nm}$. The experiment was carried out at 48,000 rpm. Differential coefficient distribution $(\mathrm{c}(\mathrm{s}))$ was fitted using SedFit 12.52. The c(s) curve suggests that BRMS1 $_{51-84}$ present a single specie with a sedimentation coefficient of $0.79 \pm 0.1$ Svedberg. (B) Equilibrium sedimentation assay was conducted with scan data acquisition at $235 \mathrm{~nm}$ and at 30, 36 and $48 \mathrm{krpm}$ of velocity, estimating an apparent molecular weight of 9,900 $\pm 30 \mathrm{Da}$. The residuals (lower panel) for the fitting between the experimental and an ideal singlespecies model (blue line) are shown.

Fig. 5: NMR characterization of BRMS1 proteins. (A) The amide and (B) methyl regions of $\mathrm{BRMS1}_{51-98}$ (top) and $\mathrm{BRMB}_{51-84}$ (bottom). Measurements were acquired at $\mathrm{pH} 7.0$ (phosphate buffer, $10 \mathrm{mM}$ ) and $298 \mathrm{~K}$. 
Fig. 6: Fluorescence changes of BRMS1 proteins. (A) Changes in the intrinsic fluorescence intensity at $315 \mathrm{~nm}$ as the $\mathrm{pH}$ was varied. (B) Changes in the fluorescence intensity of ANS at $480 \mathrm{~nm}$ as the $\mathrm{pH}$ was varied. (C) Changes in the intrinsic fluorescence intensity at selected pHs of $\mathrm{BRMS1}_{51-98}$, as the temperature was varied. The y-axis scale is arbitrary: it has been modified to allow for the comparison among the traces of the thermograms.

Fig. 7: Far-UV CD of BRMS1 proteins. (A) The far-UV CD spectra of both BRMS1s at pH 7.0. (B) The $\mathrm{pH}$-dependent changes in the $[\Theta]$ at $222 \mathrm{~nm}$. (C) Thermal denaturations, followed by the changes in ellipticity at $222 \mathrm{~nm}$, at different pHs, of BRM1 $1_{51-84}$. The y-axis scale of the raw ellipticity is arbitrary: it has been modified to allow for the comparison among the traces of the thermograms. (D) Chemical-denaturations followed by the changes in the $[\Theta]$ at $222 \mathrm{~nm}$. Measurements were carried out at $298 \mathrm{~K}$ in $10 \mathrm{mM}$ phosphate buffer $(\mathrm{pH}$ 7.0). Protein concentration used for both species was $15 \mu \mathrm{M}$ of protomer in all experiments.

Fig. 8: Superposition of BRMS1 ${ }_{51-84}$ and $B R M S 1_{51-98}$ coiled-coil motifs. Overlapping of the two chains composing both coiled coils. BRMS1 $1_{51-84}$ is shown in light brown, and BRMS1 $_{51-98}$ in light blue. The center and right images are generated upon rotation around $\mathrm{x}-$ and y-axes from their left image, and show the structural differences in the antiparallel coiled coil superposition, with a RMSD of $4.15 \AA$ using the backbone atoms. Carboxylic and amino terminals are shown as $\mathrm{Cs}$ and $\mathrm{Ns}$ (for $\mathrm{BRMS1} 1_{51-84}$ ) and $\mathrm{Cl}$ and $\mathrm{Nl}$ (in BRMS1 $1_{51-98}$ ) respectively.

Fig. 9: Accessibility of the residues of the consensus nuclear export signal (NES) in BRMS1 $_{51-98}$ structure. Detail of the residues that define the consensus NES within the hexamer conformation. The two molecules of each of the three coiled coil are represented 
with the same color and in surface representation: light brown for $\mathrm{A}-\mathrm{B}$, pink for $\mathrm{A}^{\prime}-\mathrm{B}^{\prime}$ and blue for A"-B" (' and " are A-B symmetry-related molecules). All side chains of the residues included in consensus NES (F79, L83, L86 and L88) are shown as sticks. F79, L83 and L86 occupy the positions $d, a$ and $d$ in the heptad pattern respectively, in the interacting surface of the antiparallel coiled coil, whereas L88 is located in a non-interacting $f$ position. Coordinates were obtained from PDB ID: 2XUS ${ }^{14}$.

Fig. Sup Material 1. Changes in the raw ellipticity at $222 \mathrm{~nm}$, as the TFE concentration was varied, were monitored for $B R M S 1_{51-84}$ (blank red circles) and $B R M S 1_{51-98}$ (blank blue squares). Experiments were acquired at $298 \mathrm{~K}$ in buffer phosphate $\mathrm{pH} 7.0$, and with protein concentrations of 10 and $15 \mu \mathrm{M}$ of protomer for BRMS1 $1_{51-84}$ and BRMS1 $1_{51-98}$, respectively to allow for the differences in the raw ellipticity.

Fig. Sup Material 2. Methyl (bottom) and amide (top) regions of the 1D NMR spectrum of BRMS1 $1_{51-84}$ in $80 \%$ TFE. Experiments were acquired at $298 \mathrm{~K}$, in phosphate buffer (pH 7.0).

Fig. Sup Material 3. Superposition of the BRMS1 $1_{51-98}$ hexamer and BRMS1 $1_{51-84}$ coiledcoil motif. Cartoon representation of $\mathrm{BRMS} 1_{51-98}$ quaternary structure formed by a trimer of antiparallel coiled coils packed around a ternary axis ${ }^{14}$. The BRMS1 $1_{51-84}$ coiled coil is shown in orange and the two molecules of BRMS1 ${ }_{51-98}$ coiled coil are represented in blue. Two of the main residues implicated in the formation of the hexamer by interacting through the N-termini of the chains (Arg56 and Arg57) are represented as sticks in yellow. The corresponding residues in BRMS1 $1_{51-84}$ coiled coil are colored in red. Other view of the superposition of both coiled-coil motifs and the two neighboring chains with which are interacting through the Nterminus in the hexamer, is shown in the boxed panel. Carboxylic and amino terminals of $\mathrm{BRMS1}_{51-98}$ are labeled $\mathrm{Cl}$ and $\mathrm{Nl}$ respectively, and Ns represents the amino-terminal of BRMS1 $1_{51-84}$ 


\section{TABLES}

Table 1. Data collection and refinement statistics for BRMS1 $1_{51-84}$ structure.

${ }^{\mathrm{a}} \mathrm{R}_{\text {meas }}=\left\{\Sigma_{h k l}[\mathrm{~N} /(\mathrm{N}-1)]^{1 / 2} \Sigma_{\mathrm{i}} \mid I_{i}(h k l)-\langle I(h k l)>|\right\} / \Sigma_{h k l} \Sigma_{\mathrm{i}} I_{i}(h k l)$, where $I_{i}(h k l)$ are the observed intensities, $<I(h k l)>$ are the average intensities and $\mathrm{N}$ is the multiplicity of reflection $h k l$.

${ }^{\mathrm{b}} \mathrm{R}_{\mathrm{pim}}=\left\{\Sigma_{h k l}[1 /(\mathrm{N}-1)]^{1 / 2} \Sigma_{\mathrm{i}}\left|I_{i}(h k l)-<I(h k l)>\right|\right\} / \Sigma_{h k l} \Sigma_{\mathrm{i}} I_{i}(h k l)$, where $I_{i}(h k l)$ are the observed intensity, $<I(h k l)>$ are the average intensities and $\mathrm{N}$ is the multiplicity of reflection $h k l$.

${ }^{c}$ Mean $[I / \sigma(I)]$ is the average of the relation between the intensity of the diffraction and the background.

${ }^{\mathrm{d}} \mathrm{R}_{\text {factor }}=\Sigma_{h k l}\left\{\left[F_{\text {obs }}(h k l)\right]-\left[F_{\text {calc }}(h k l)\right]\right\} / \Sigma_{h k l}\left[F_{o b s}(h k l)\right]$, where $F_{o b s}(h k l)$ and $F_{\text {calc }}(h k l)$ are the structure factors observed and calculated, respectively.

${ }^{\mathrm{e}} \mathrm{R}_{\text {free }}$ corresponds to $\mathrm{R}_{\text {factor }}$ calculated using $5 \%$ of the total reflections selected randomly and excluded during refinement.

${ }^{\mathrm{f}} \mathrm{RMSD}$ is the root mean square deviation. 


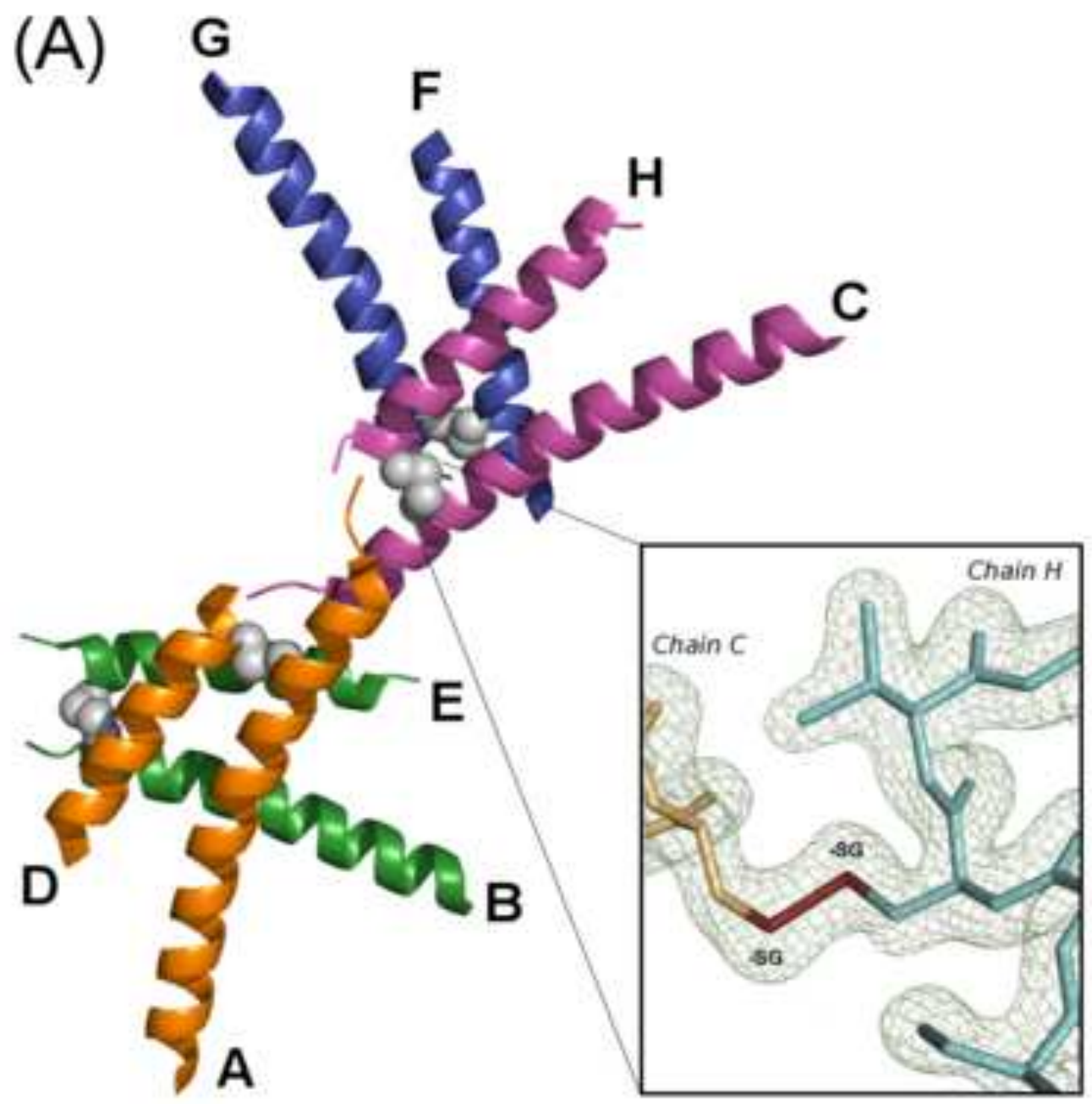

(B) $C_{\text {sym } 5}$

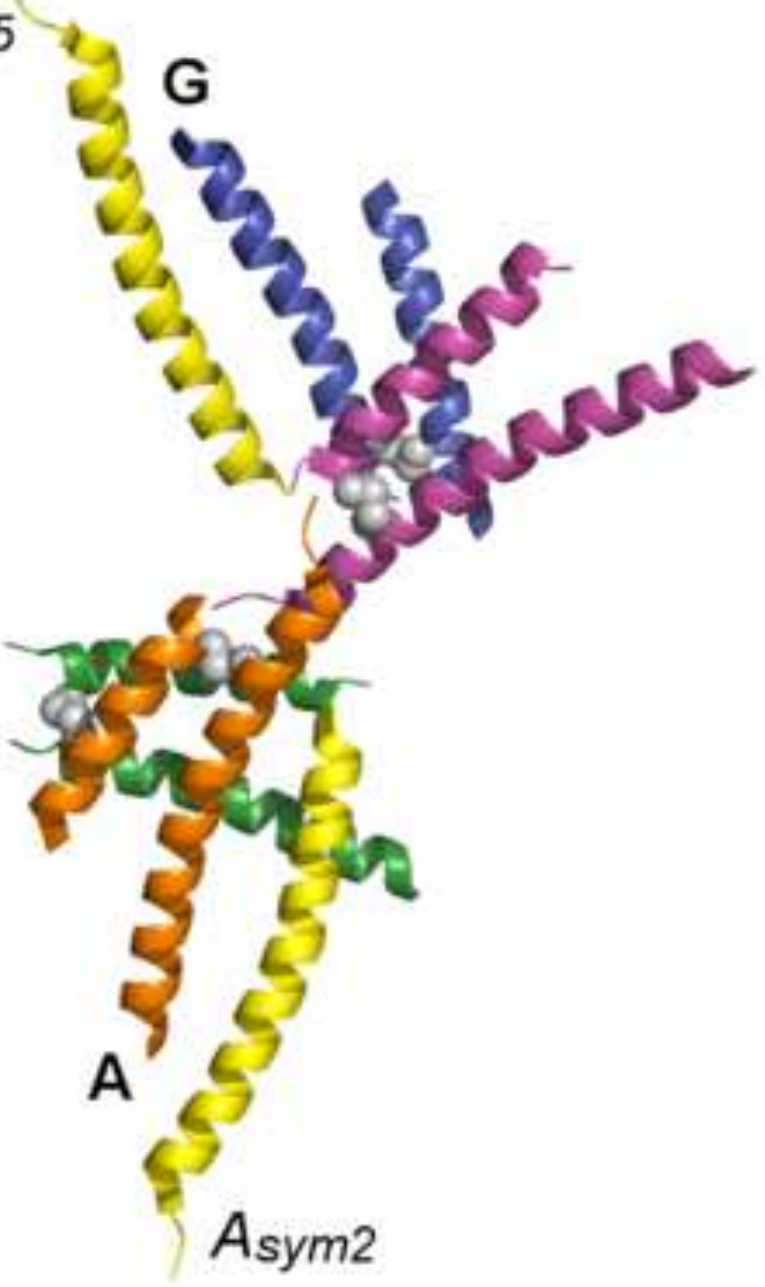


Figure 2
Click here to download high resolution image

(A)

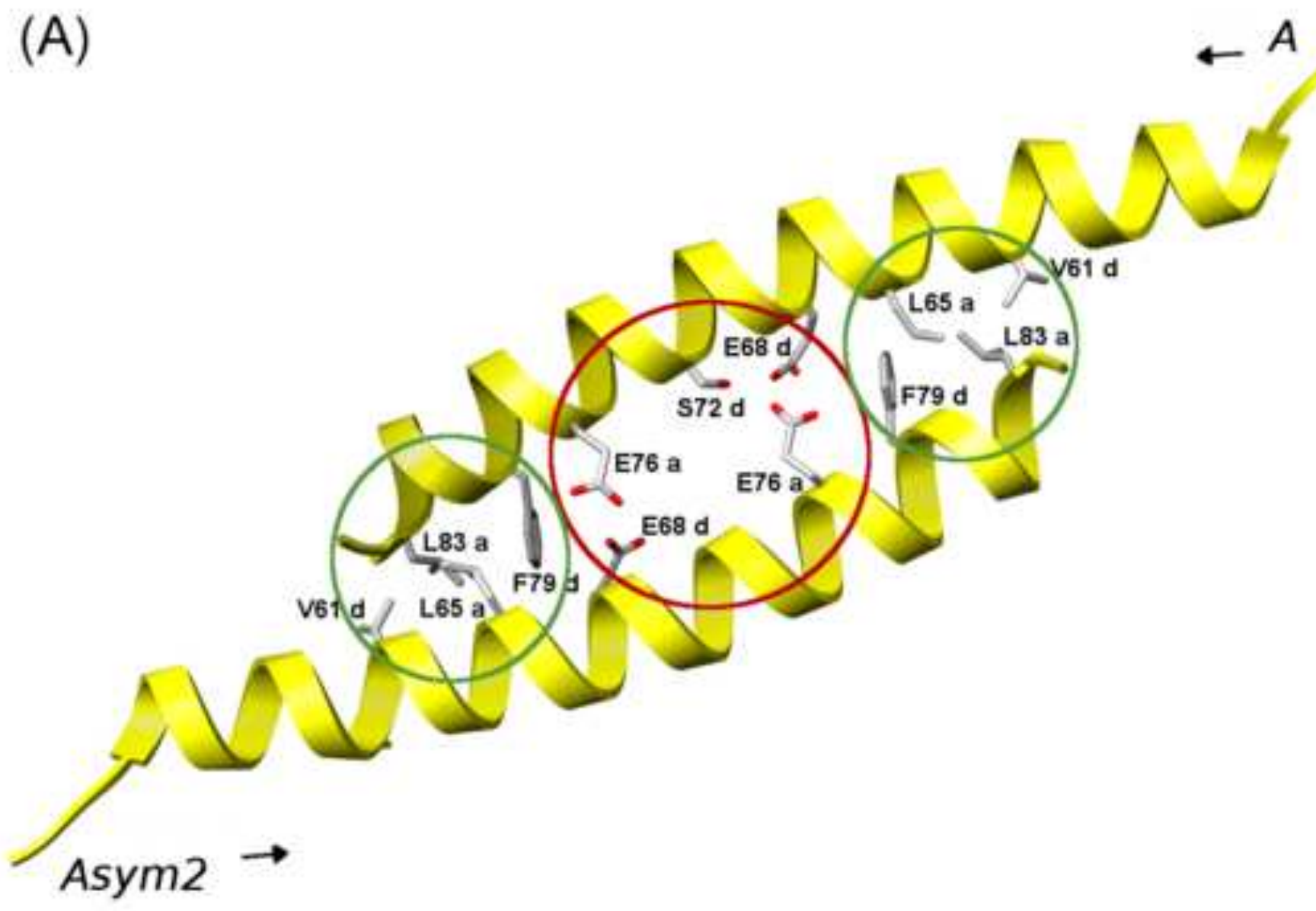

(B)

Asym2 $\rightarrow$

Asym2

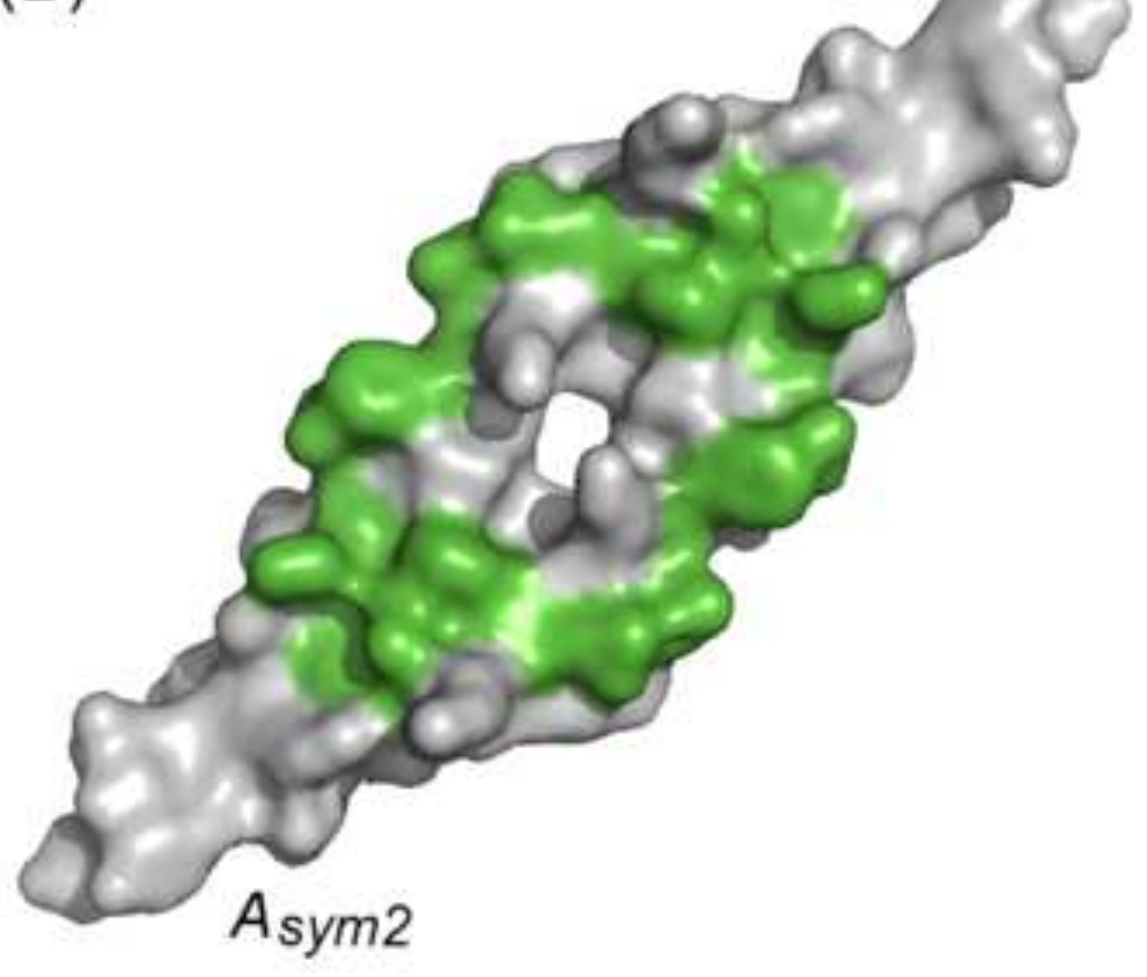

A A 
Figure 3

Click here to download high resolution image

(A)

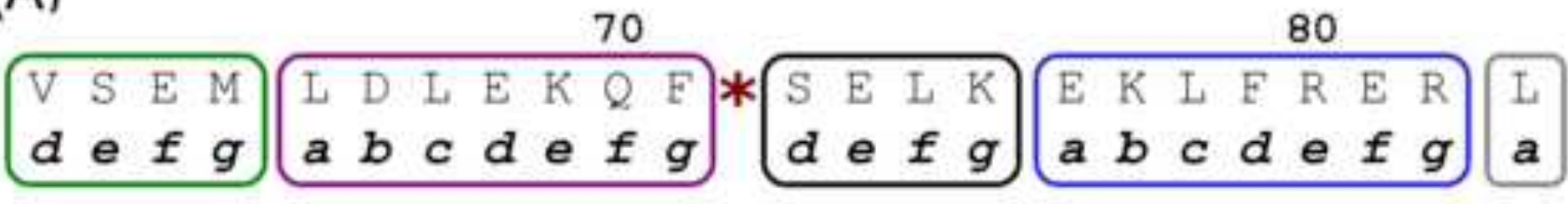

(B)

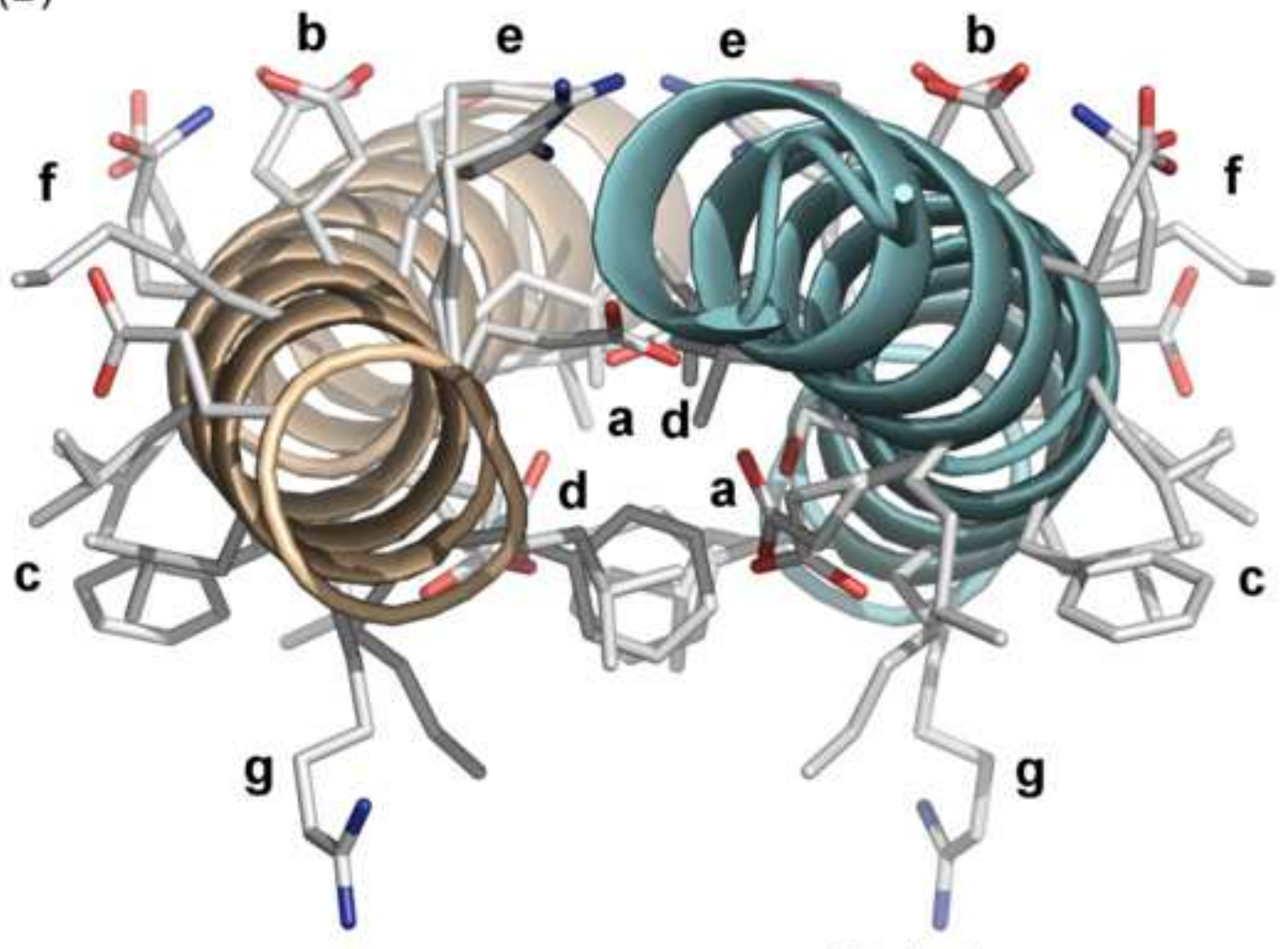

Chain A

Chain A sym2 
(A)

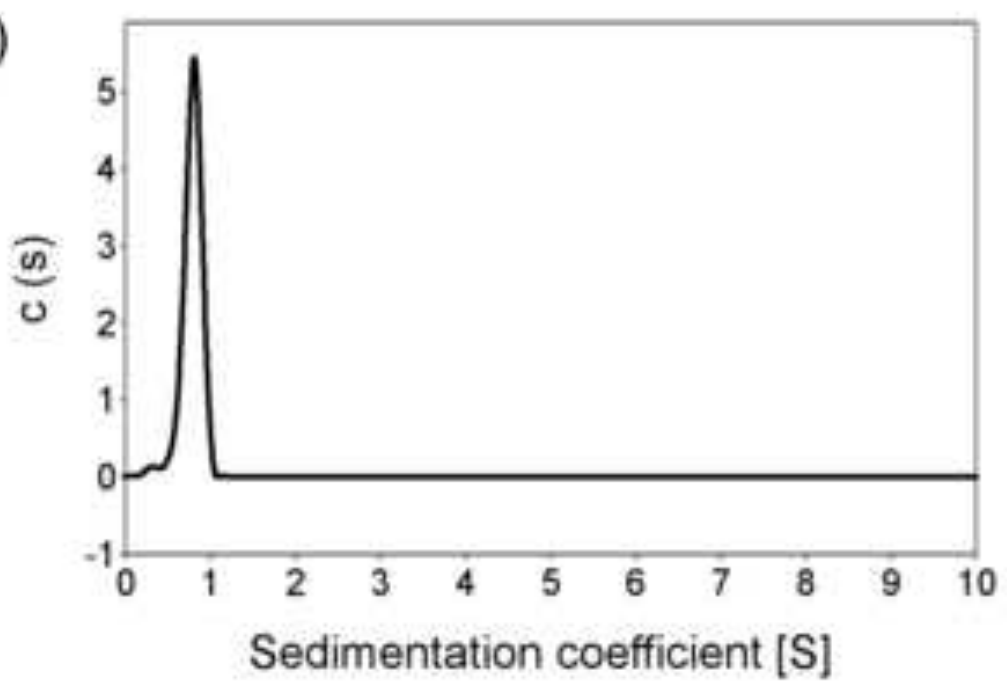

(B)
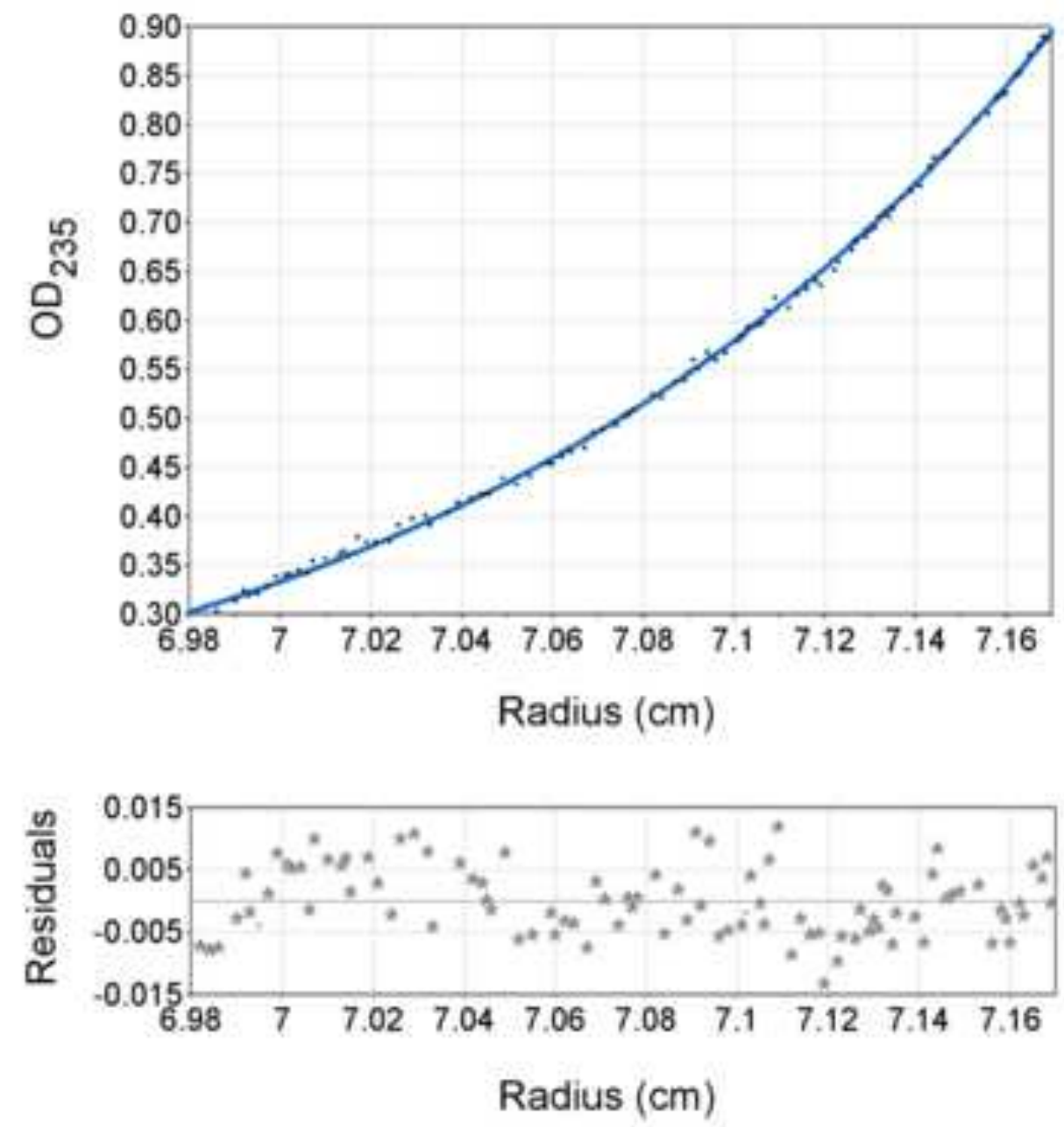
Click here to download high resolution image
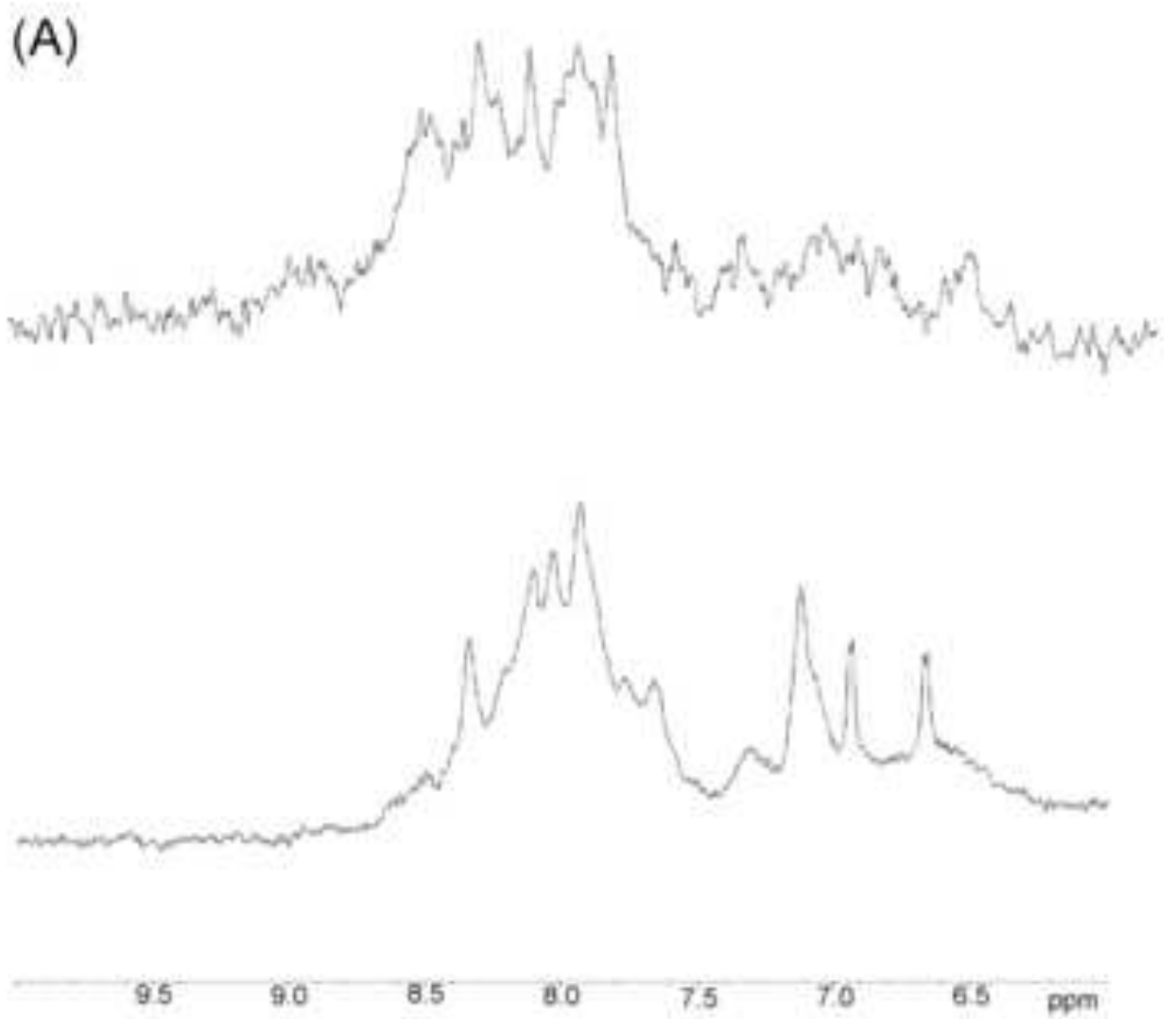

(B)
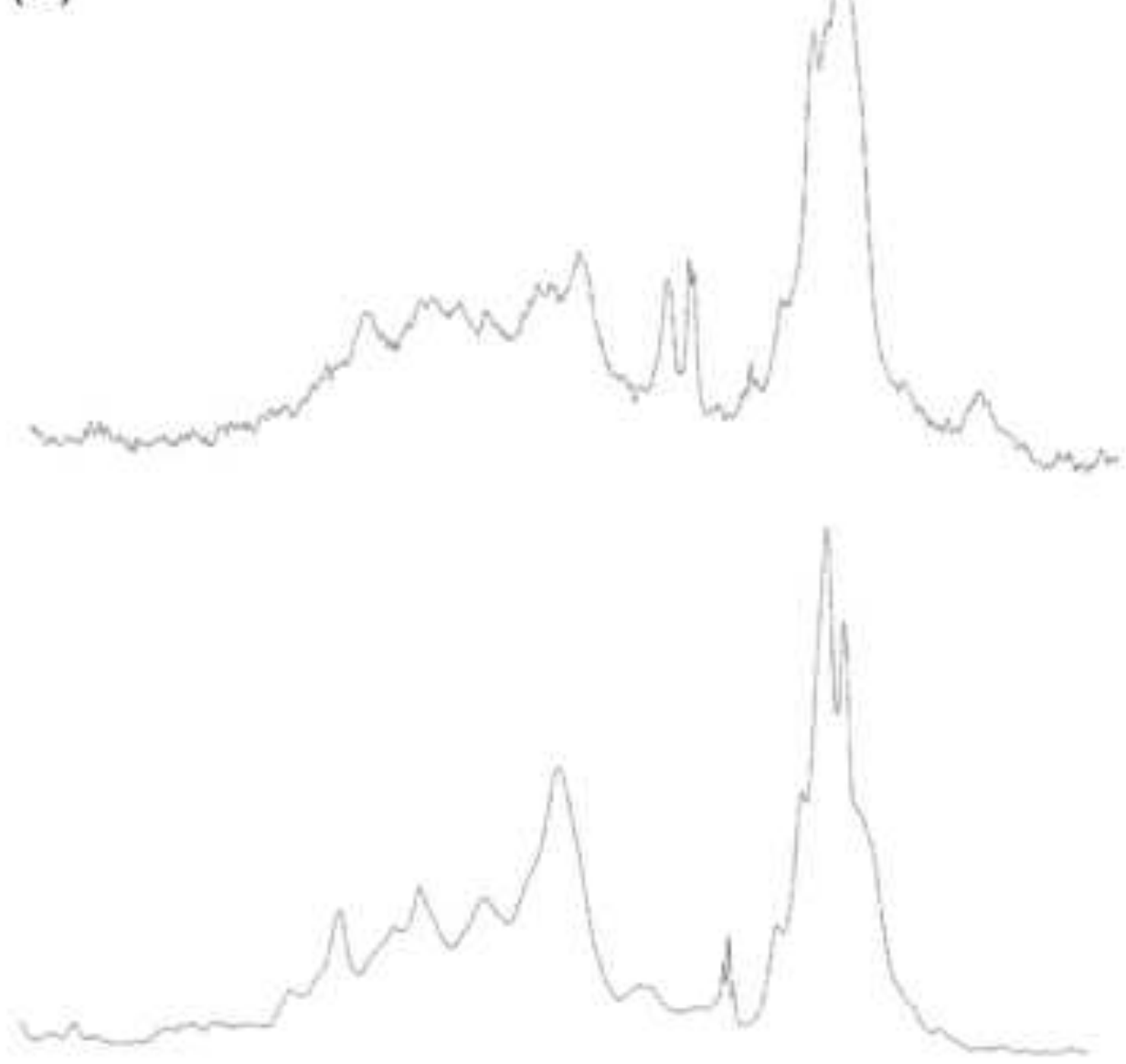

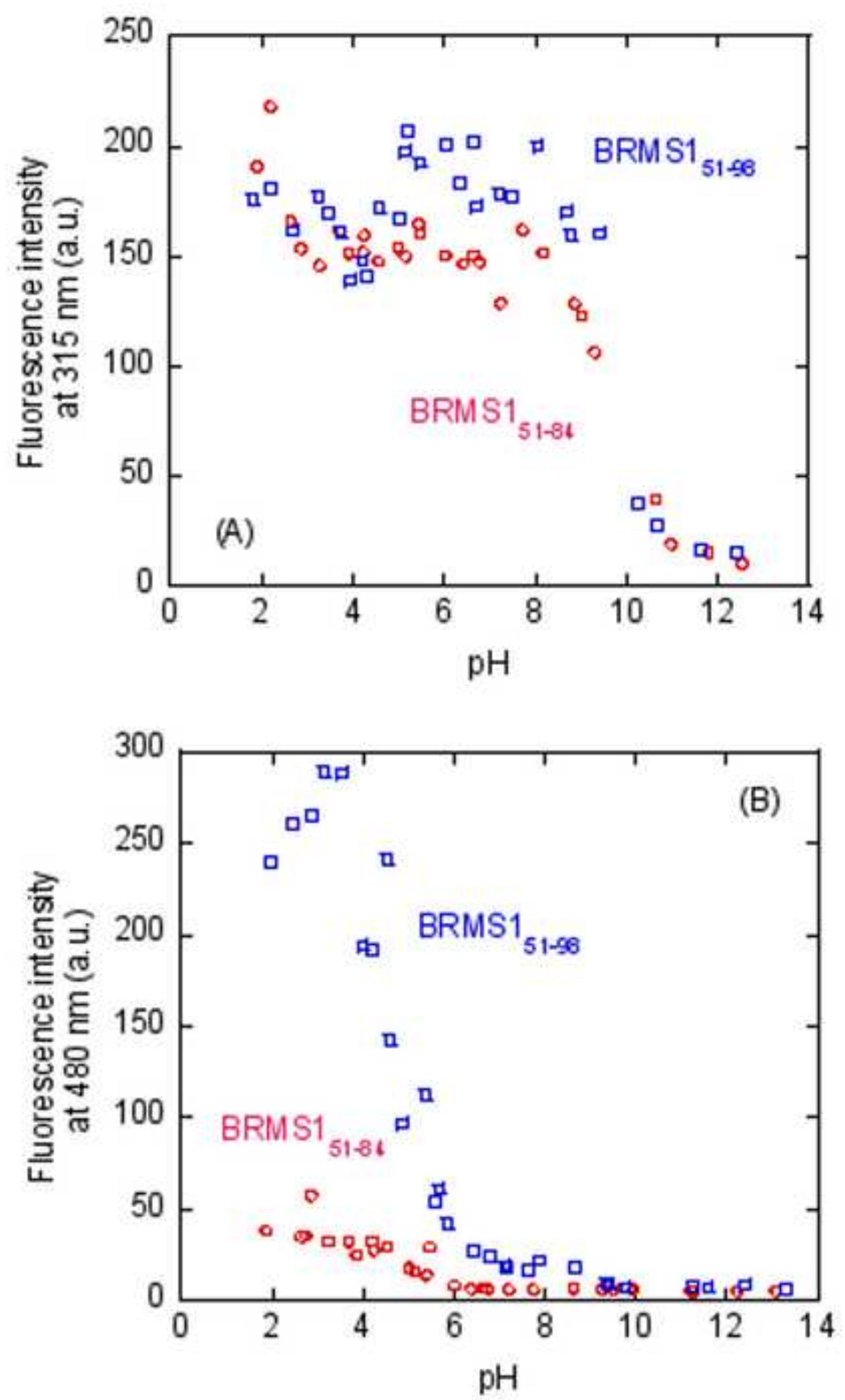

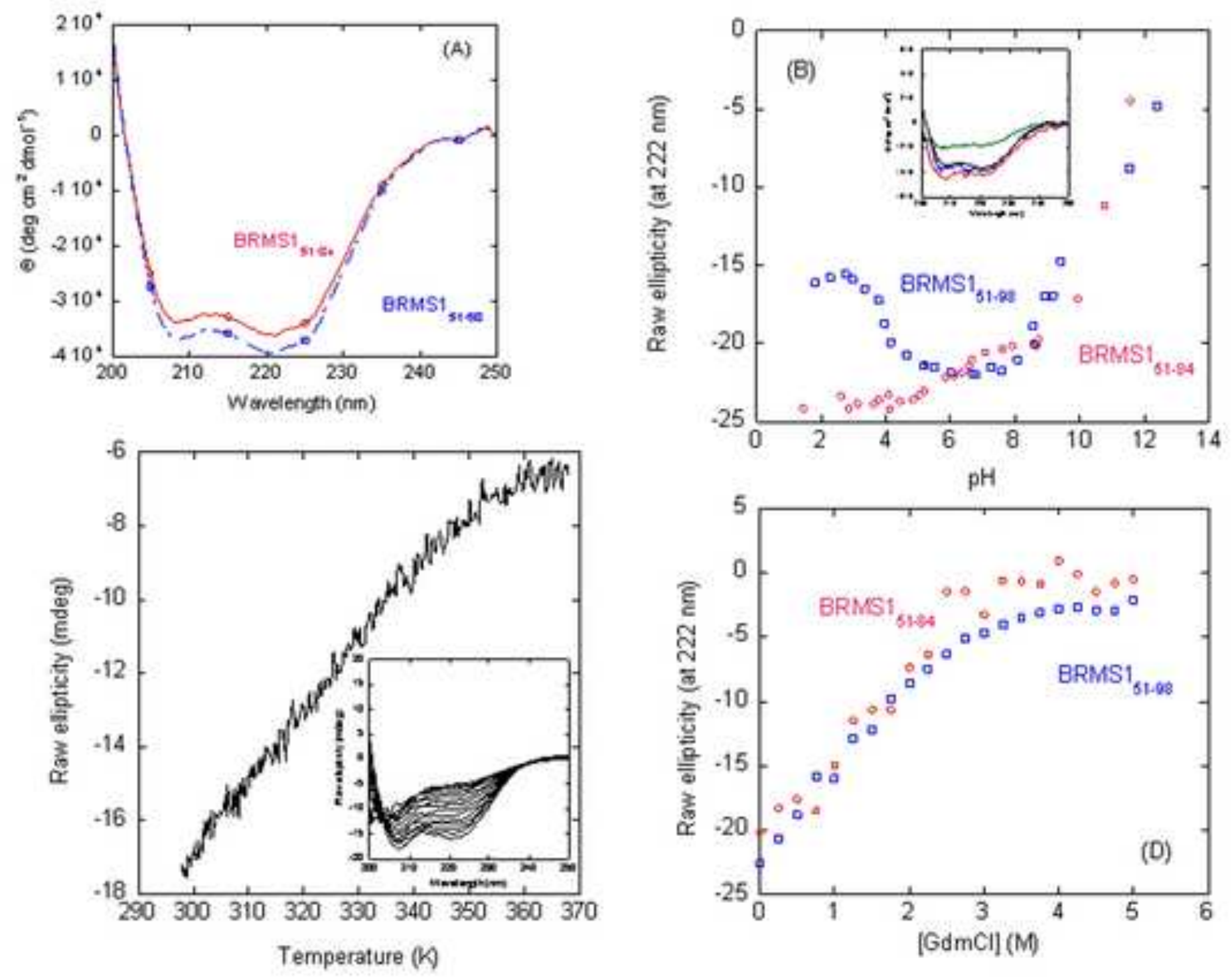
Figure 8

Click here to download high resolution image

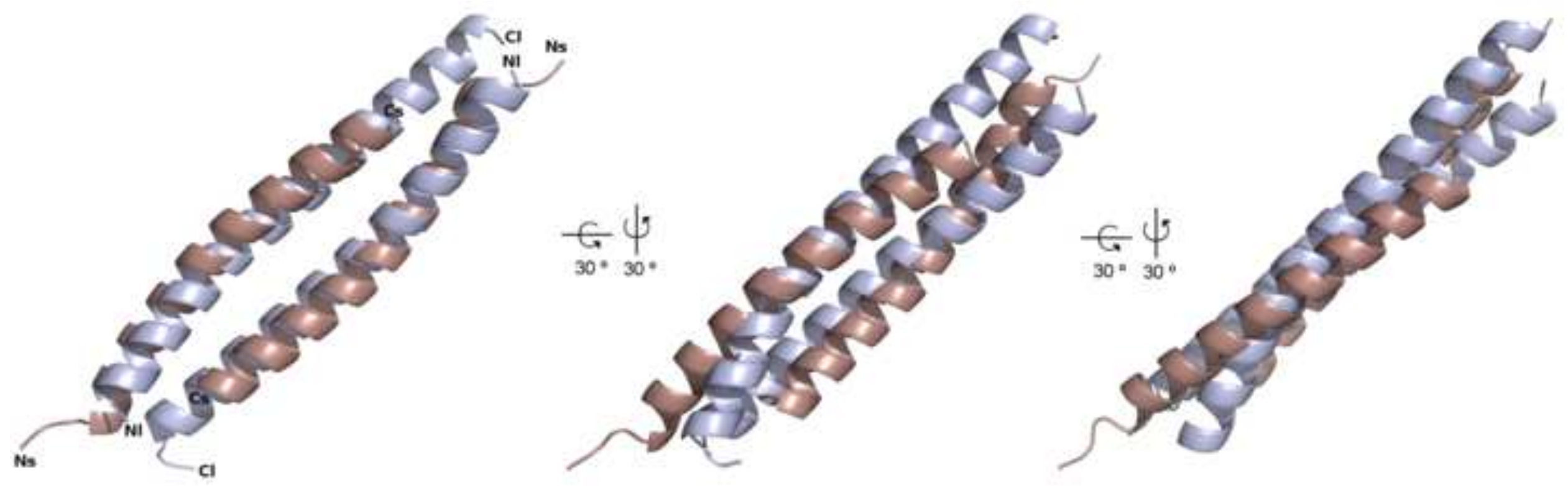


Table 1.

BRMS1 $_{51-84}$

\section{Data collection}

Native

Space group

Unit Cell Dimensions

a, b, c $(\AA)$

$\alpha, \beta, \gamma\left({ }^{\circ}\right)$

Resolution (last shell) $(\AA)$

Observations (unique)

Completeness (\%) (last shell)

Multiplicity (last shell)

$\mathrm{R}_{\text {meas }}{ }^{\mathrm{a}}$ (last shell)

$\mathrm{R}_{\text {pim }}{ }^{\mathrm{b}}$ (last shell)

Mean $[I / \sigma(I)]^{\mathrm{c}}$ (last shell)

Anomalous completeness (\%)

Anomalous multiplicity

Anomalous signal
$C 222_{1}$

42.64, 191.27, 71.94

90, 90,90

$95.56-2.00(2.11-2.00) \quad 95.78-2.20(2.32-2.20)$

$146,108(20,344)$

$99.2(94.7)$

$7.2(3.9)$

$0.055(0.237)$

$0.019(0.119)$

$28.2(7.2)$

\section{Derivative (Hg)}

$C 222_{1}$

$42.41,191.40,71.87$

90, 90,90

$108,281 \quad(15,378)$

$100.0(100.0)$

$7.0(7.2)$

$0.106(0.577)$

$0.041(0.214)$

15.4 (3.7)

99.9

3.7

1.168

\begin{tabular}{ll}
\hline $\begin{array}{l}\text { Refinement and validation } \\
\text { Non-hydrogen atoms (solvent molecules) }\end{array}$ & 2,000 (153) \\
Heteroatoms & 1 \\
$\mathrm{SO}_{4}$ & 1 \\
$\mathrm{Cl}$ & 1 \\
$\left.\mathrm{Acetate}_{\mathrm{d}} \mathrm{R}_{\text {free }}^{\mathrm{e}}\right)(\%)$ & $19.2(25.8)$ \\
$\mathrm{R}_{\text {work }}$ & 0.019 \\
$\mathrm{RMSD}^{\mathrm{f}}$ bond length $(\AA)$ & 2.061 \\
$\mathrm{RMSD}^{\mathrm{f}}$ bond angles $\left({ }^{\mathrm{O}}\right)$ & \\
$\mathrm{B}$ factor $\left(\AA^{2}\right)$ & 38.76 \\
$\quad$ main/side chain & 45.52 \\
$\quad$ solvent & 40.77 \\
heteroatoms & \\
Ramachandran $(\%)$ & 98.96 \\
$\quad$ favoured region & 0.52
\end{tabular}


Click here to download Electronic Supplementary Material (online publication only): Fig1SMallver.tif 
Fig2SMallver.tif
Click here to dov

Click here to download Electronic Supplementary Material (online publication only): Fig2SMallver.tif

\begin{abstract}
Click here to
\end{abstract}



Fig3SM_L.tiff
Click here to download Electronic Supplementary Material (online publication only): Fig3SM_L.tiff Fig3SM_L.tiff
Click here to download Electronic Supplementary Material (online publication only): Fig3SM_L.tiff Click here to download Electronic Supplementary Material (online publication only): Fig3SM_L.tiff (1) (1) (1) $x^{2}$ .

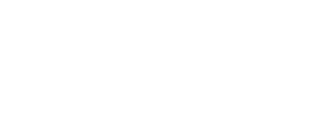
. . . . . . . . .

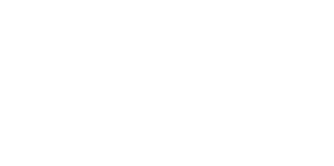
. .

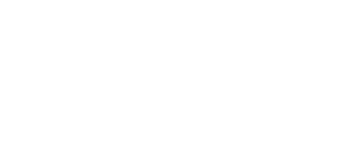
. . (1) (1) (1)

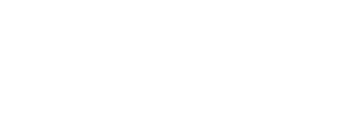
(1) (

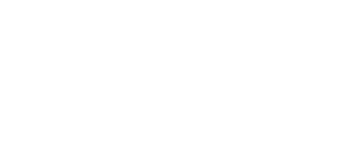
(1) 
Click here to download Electronic Supplementary Material (online publication only): Fig4SM_L.tiff Fig4SM_L.tiff
Click here to

作 (1) Click here to download Electronic Supplementary Material (online publication only): Fig4SM_L.tiff (1)

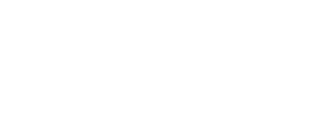

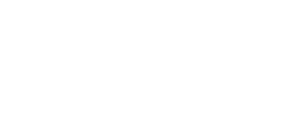
.

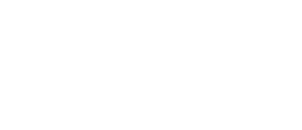

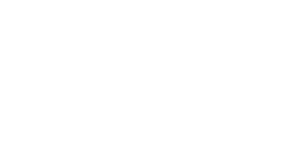
. . . . 列 . . 列 . . . . . . . . . 

Fig5SM.tif
Click here to download Electronic Supplementary Material (online publication only): Fig5SM.tif

if

